Publ. Mat. 62 (2018), 355-396

DOI: 10.5565 /PUBLMAT6221803

\title{
HEEGNER POINTS ON \\ HIJIKATA-PIZER-SHEMANSKE CURVES AND THE BIRCH AND SWINNERTON-DYER CONJECTURE
}

\author{
Matteo longo, Víctor Rotger, and Carlos de Vera-Piquero
}

\begin{abstract}
We study Heegner points on elliptic curves, or more generally modular abelian varieties, coming from uniformization by Shimura curves attached to a rather general type of quaternionic orders. We address several questions arising from the Birch and Swinnerton-Dyer (BSD) conjecture in this general context. In particular, under mild technical conditions, we show the existence of non-torsion Heegner points on elliptic curves in all situations in which the BSD conjecture predicts their existence.
\end{abstract}

2010 Mathematics Subject Classification: 11G05, 11G18, 11G40.

Key words: BSD conjecture, Heegner points, $L$-functions, Shimura curves.

\section{Introduction}

Let $E / \mathbb{Q}$ be an elliptic curve of conductor $N$ over the field of rational numbers. Let $K$ be an imaginary quadratic field of discriminant $-D$ and

$$
\chi: G_{K}=\operatorname{Gal}(\bar{K} / K) \longrightarrow \mathbb{C}^{\times}
$$

be a character of finite order of the absolute Galois group of $K$. We assume throughout that $\chi$ is anticyclotomic, meaning that $\chi\left(\tau \sigma \tau^{-1}\right)=$ $\chi^{-1}(\sigma)$ for any $\sigma \in G_{K}$ and $\tau \in G_{\mathbb{Q}} \backslash G_{K}$.

The abelian extension cut out by $\chi$ is a ring class field associated to some order $R_{c}$ in $K$ of conductor $c=c(\chi) \geq 1$. Let $H_{c}$ denote

During the work on this paper, M. Longo was partially supported by PRIN 2010/11 "Arithmetic Algebraic Geometry and Number Theory" and PRAT 2013 "Arithmetic of Varieties over Number Fields"; V. Rotger was partially supported by Grant MTM2015-63829-P; and C. de Vera-Piquero was partially supported by Grant MTM2015-63829-P and by the German Research Council via SFB/TR 45. This project has received funding from the European Research Council (ERC) under the European Union's Horizon 2020 research and innovation programme (Grant Agreement No 682152). The second and third authors also acknowledge financial support from the Spanish Ministry of Economy and Competitiveness, through the María de Maeztu Programme for Units of Excellence in R\&D (MDM-2014-0445). 
the corresponding abelian extension, determined by the isomorphism $\operatorname{Gal}\left(H_{c} / K\right) \simeq \operatorname{Pic}\left(R_{c}\right)$ induced by the Artin map. Define the $\chi$-isotypical component of the Mordell-Weil group of $E$ over $H_{c}$ as

$$
E\left(H_{c}\right)^{\chi}:=\left\{x \in E\left(H_{c}\right) \otimes \mathbb{C}: \sigma(x)=\chi(\sigma) x, \forall \sigma \in \operatorname{Gal}\left(H_{c} / K\right)\right\},
$$

and let $L(E / K, \chi, s)$ denote the Rankin $L$-series associated to the twist of $E / K$ by $\chi$. Since $\chi$ is anticyclotomic, the motive associated to $L(E / K, \chi, s)$ is Kummer self-dual and this implies that the global root number $\varepsilon(E / K, \chi)$ of $L(E / K, \chi, s)$ is either +1 or -1 . Assume for the remainder of the article that

$$
\varepsilon(E / K, \chi)=-1,
$$

hence in particular $L(E / K, \chi, s)$ vanishes at the central critical point $s=$ 1 .

In this situation, the Galois equivariant version of the Birch-Swinnerton-Dyer conjecture predicts that the implication

$$
L^{\prime}(E / K, \chi, 1) \neq 0 \stackrel{?}{\Longrightarrow} \operatorname{dim}_{\mathbb{C}} E\left(H_{c}\right)^{\chi}=1
$$

holds true for all triplets $(E, K, \chi)$ as above.

The well-known strategy for proving this implication was established by the pioneer works of Gross-Zagier $[\mathbf{G Z}]$ and Kolyvagin $[\mathbf{K o l}]$, and consists in exploiting the Euler system of Heegner points on $E$ arising from classical modular parametrisations. Since the breakthrough of Gross, Zagier, and Kolyvagin in the late eighties, this method was generalized by Zhang and his school ([Zha], [YZZ]) by extending the range of modular parametrisations of the elliptic curve using Shimura curves associated to orders in quaternion algebras. As described in [Nek1], these ideas have been turned into a machinery which allows one to prove (1) for a given triplet $(E, K, \chi)$, subject to the existence of non-torsion Heegner points in $E\left(H_{c}\right)$.

Unfortunately, however, the existing literature does not make explicit the Shimura uniformization in all scenarios in which (1) holds. Indeed, this is established in the afore mentioned works under so-called Heegner hypotheses which typically leave aside many cases in which there is a prime $p$ dividing both $N$ and $D c$. As pointed out, the reason may be traced to the difficulty of proving the existence of non-torsion Heegner points in the Mordell-Weil group of $E$ over the abelian extension $H_{c}$ of $K$ cut out by $\chi$.

Recent and interesting progress has been recently made on such settings by Kohen-Pacetti [KP1], [KP2] and Cai-Chen-Liu in [CCL], where the modular parametrisation is afforded by a classical modular curve attached to a Cartan level structure in the split matrix algebra. 
Nevertheless, there are still many cases of (1) in which a Shimura curve uniformization is not made explicit by these works. The simplest scenario not covered by previous works arises when $E / \mathbb{Q}$ is an elliptic curve of conductor $N=p^{2} q$, where $p$ and $q$ are two distinct odd primes such that $p$ is ramified and $q$ is inert in $K$. In this setting it may perfectly be the case that the sign of the functional equation satisfied by $L(E / K, s)$ be -1 (indeed, this holds under certain arithmetic conditions on the local root numbers of the functional equation of $L(E / K, s)$ at $p$ and $q$ which are made precise below), and although $[\mathbf{Y Z Z}]$ proves the existence of a Heegner system in some Shimura curve, the desired uniformization is not made explicit. It is the purpose of this paper to describe explicitly the optimal Shimura curve uniformization, hence this particularly simple example can be thought as a motivation for this work.

The main goal we set ourselves for this project is covering this gap in the literature by providing a result proving (1) for all triplets $(E, K, \chi)$, with no additional hypothesis, and proving the existence of non-trivial Heegner points on the field cut out by $\chi$. As the reader will realize, we fall short at achieving this goal, but we prove a fairly general theorem encompassing all previously known results and covering all possible scenarios allowed by (1) but for a few parasitic ones.

We do that by considering Jacobians $\operatorname{Jac}\left(X_{U}\right)$ of Shimura curves $X_{U}$ associated to a rather general collection of non-maximal compact open subgroups $U \subseteq \hat{B}^{\times}=(B \otimes \hat{\mathbb{Z}})^{\times}$in indefinite quaternion algebras $B / \mathbb{Q}$; these correspond to orders defined by local conditions of Eichler or Cartan type when the quaternion algebra is split, and to orders introduced by Pizer [Piz2] and Hijikata-Pizer-Shemanske [HPS1] in the non-split case.

Let us state our theorem more precisely and describe the structure of this paper. Section 1 is devoted to introduce an explicit family of special orders $\mathcal{R}$ in $B$ which shall play a central role in our work. These orders are determined by local data at the primes of bad reduction of the elliptic curve $E$, following classical work of Hijikata, Pizer, and Shemanske that apparently did not receive the attention it justly deserved. As a piece of notation, for any order $\mathcal{R}$ of $B$ we let $X_{\mathcal{R}}$ denote the Shimura curve associated to $U=\hat{\mathcal{R}}^{\times}=(\mathcal{R} \otimes \hat{\mathbb{Z}})^{\times}$. Also, to emphasize the role of $B$, we will sometimes write $X_{B, \mathcal{R}}$ for $X_{\mathcal{R}}$.

In Section 2 we study the Shimura curves $X_{\mathcal{R}}$ associated to the above mentioned Hijikata-Pizer-Shemanske orders and work out explicitly the Jacquet-Langlands correspondence for these curves, which allows us to dispose of a rich source of modular parametrizations of the elliptic curve $E$. For any integer $c$, there is a (possibly empty) collection of 
distinguished points on $X_{\mathcal{R}}$, called Heegner points of conductor $c$. The set of Heegner points of conductor $c$ on $X_{\mathcal{R}}$ is in natural correspondence with the set of conjugacy classes of optimal embeddings of the quadratic order $R_{c}$ in the quaternion order $\mathcal{R}$, and we denote it $\operatorname{Heeg}(\mathcal{R}, K, c)$. We say that a point in $E\left(H_{c}\right)$ is a Heegner point of conductor $c$ associated to the order $\mathcal{R}$ if it is the image of a Heegner point of conductor $c$ in $X_{\mathcal{R}}$ for some uniformization map

$$
\pi_{E}: \operatorname{Jac}\left(X_{\mathcal{R}}\right) \longrightarrow E
$$

defined over $\mathbb{Q}$. The corresponding set of Heegner points is denoted $\operatorname{Heeg}_{E}(\mathcal{R}, K, c)$.

In Section 3 we perform a careful and detailed analysis of the rather delicate and involved theory of optimal embeddings of quadratic orders into Hijikata-Pizer-Shemanske orders. Combining all together this allows to prove the main result of this article. A slightly simplified version of this result is the following. The main virtue of the statement below with respect to previous results available in the literature is that it is both general (removing nearly all unnecessary hypothesis on divisibility and congruence relations among $N, D$, and $c$ ) and precise (pointing out to a completely explicit Shimura curve).

Theorem A. Let $E / \mathbb{Q}$ be an elliptic curve of conductor $N$ not divisible neither by $2^{3}$ nor by $3^{3}$, and suppose that the newform $f \in S_{2}\left(\Gamma_{0}(N)\right)$ attached to $E$ by modularity is primitive. Let $K$ be an imaginary quadratic field of discriminant $-D$ and $\chi$ be an arbitrary anticyclotomic character of conductor $c \geq 1$. Assume that $\varepsilon(E / K, \chi)=-1$. Then

(1) There exists an explicit Hijikata-Pizer-Shemanske order $\mathcal{R}=$ $\mathcal{R}(E, K, \chi)$ for which the set of Heegner points $\operatorname{Heeg}_{E}(\mathcal{R}, K, c)$ in $E\left(H_{c}\right)$ is non-empty.

(2) If $L^{\prime}(E / K, \chi, 1) \neq 0$ and $E$ does not acquire $C M$ over any imaginary quadratic field contained in $H_{c}$, then $\operatorname{dim}_{\mathbb{C}}\left(E\left(H_{c}\right) \otimes \mathbb{C}\right)^{\chi}=1$.

The condition that $f$ is primitive means here that $f$ is not a twist of a modular form of level $M<N$ (cf. Definition 4.18). Theorem A is proved in the last section of the article, where we also provide a more general but more involved statement (see Theorem 4.16), in which we discuss the cases when $2^{3}$ or $3^{3}$ divide $N$, and show also that the primitivity assumption is only needed locally at some primes dividing $N$. We also prove a similar but weaker result for modular abelian varieties in Theorem 4.5, and we close the paper with a conjecture on the existence of Heegner points on modular abelian varieties. 
Statement (2) in the above theorem follows from (1) and well-known Kolyvagin type arguments which are spelled out in detail in [Nek1]. Namely, given

- a parametrization of the elliptic curve $E$ by a Shimura curve $X_{B, U}$,

- a Heegner point $x$ in $X_{B, U}\left(K^{\mathrm{ab}}\right)$, rational over a subfield $K(x) \subseteq$ $K^{\mathrm{ab}}$, and

- a character $\chi$ factoring through $\operatorname{Gal}(K(x) / K)$,

Nekovár shows that if the special value of the derivative of the complex $L$-function at $s=1$ is nonzero, then the dimension of the $\mathbb{C}$-vector space $(E(K(x)) \otimes \mathbb{C})^{\chi}$ is equal to 1 , provided that $E$ does not acquire CM over any imaginary quadratic field contained in $K(x)^{\operatorname{ker}(\chi)}$.

In some sense, our Theorem A reverses the logical order of the result in [Nek1], starting with a character of a given conductor and asking for a Heegner point rational over the subextension of $K^{\text {ab }}$ cut out by that character. Therefore, the whole focus of our work is on statement (1) of the above theorem. More precisely, this work grows out from a systematic study of existence conditions for Heegner points in all scenarios in which the BSD conjecture predicts the existence of a non-zero element in $\left(E\left(H_{c}\right) \otimes \mathbb{C}\right)^{\chi}$. To understand the flavour of this work, it is therefore important to stress that we do not require any condition on the triplet $(N, D, c)$, besides the above restrictions at 2 and 3 (cf. also Assumption 4.9). Quite surprisingly, the interplay between local root numbers, non-vanishing of the first derivative of the $L$-function and the theory of optimal embeddings shows that these conditions match perfectly and, in all relevant cases, Heegner points do exist.

One of the main motivations that led us to work on this project is that these curves can be $p$-adically uniformized by the $p$-adic rigid analytic space corresponding to the first (abelian) covering of the Drinfel'd tower over the $p$-adic upper half plane $\mathcal{H}_{p}$. This rigid analytic space has an explicit description (see [Tei]) which can be used to study $p$-adic aspects of Heegner points, including their connection to Iwasawa theory and $p$-adic $L$-functions, as in the case where the elliptic curve has multiplicative reduction and can be uniformized by Drinfel'd upper half plane (cf. [BD1], [BD2], [BD3]).

It would also be highly interesting to extend the theory of Stark-Heegner points in this context (starting with the foundational paper [Dar], and developed in several subsequent works). Such a generalization is however not straight-forward, essentially because the Jacobian varieties of the Shimura curves referred to above have additive reduction at $p$ (as opposed to having toric reduction, which is a crucial feature in the above 
approaches). We regard this as an exciting obstacle to overcome rather than a forbidding difficulty, and this note aims to settle the first step towards this program that we hope to pursue in the near future.

Acknowledgements. We thank the anonymous referee for very valuable comments and suggestions that have helped to improve the exposition of this paper.

\section{Shimura curves}

Let $\hat{\mathbb{Z}}$ denote the profinite completion of $\mathbb{Z}$, and write $\hat{R}:=R \otimes_{\mathbb{Z}} \hat{\mathbb{Z}}$ for every $\mathbb{Z}$-algebra $R$. Fix an integer $\Delta>1$, which is assumed to be squarefree and the product of an even number of primes, and let $B$ be the indefinite rational quaternion algebra of reduced discriminant $\Delta$. Write $\hat{B}=B \otimes_{\mathbb{Q}} \hat{\mathbb{Q}}$ for the finite adelization of $B$. We also fix a maximal order $\mathcal{O}$ in $B$; recall that such an order is unique up to conjugation by an element in $B^{\times}$. Finally, we shall fix an isomorphism $B_{\infty}:=B \otimes_{\mathbb{Q}} \mathbb{R} \rightarrow \mathrm{M}_{2}(\mathbb{R})$, under which $B^{\times}$might be seen as a subgroup of $\mathrm{GL}_{2}(\mathbb{R})$.

1.1. Shimura curves. Let $\mathcal{H}^{ \pm}=\mathbb{C}-\mathbb{R}=\mathbb{P}^{1}(\mathbb{C})-\mathbb{P}^{1}(\mathbb{R})$ be the union of the upper and lower complex half planes, which might be identified with the set of $\mathbb{R}$-algebra homomorphisms $\operatorname{Hom}\left(\mathbb{C}, \mathrm{M}_{2}(\mathbb{R})\right)$. The action of $B^{\times}$by linear fractional transformations on $\mathcal{H}^{ \pm}$corresponds under this identification to the action of $B^{\times}$by conjugation on $\operatorname{Hom}\left(\mathbb{C}, \mathrm{M}_{2}(\mathbb{R})\right)$.

For any pact open subgroup $U$ of $\hat{\mathcal{O}}^{\times}$, one can consider the topological space of double cosets

$$
X_{U}=\left(U \backslash \hat{B}^{\times} \times \operatorname{Hom}\left(\mathbb{C}, \mathrm{M}_{2}(\mathbb{R})\right)\right) / B^{\times},
$$

where notice that $U$ acts naturally on $\hat{B}^{\times}$by multiplication on the left and $B^{\times}$acts both on $\hat{B}^{\times}$(diagonally) and on $\operatorname{Hom}\left(\mathbb{C}, \mathrm{M}_{2}(\mathbb{R})\right)$. By the work of Shimura and Deligne (cf., e.g. [Shi], [Del1]), $X_{U}$ admits the structure of an algebraic curve over $\mathbb{Q}$ and a canonical model, which we shall still denote by $X_{U} / \mathbb{Q}$. This will be referred to as the Shimura curve associated with $U$.

Although $X_{U}$ is connected over $\mathbb{Q}$, it might not be in general geometrically connected. Indeed, the set of geometric connected components of $X_{U}$ (that is, the set of connected components of $\bar{X}_{U}:=X_{U} \times \mathbb{Q} \overline{\mathbb{Q}}$ ) is identified with the finite set of double cosets $U \backslash \hat{B}^{\times} / B^{\times}$. Such components are defined over an abelian extension of $\mathbb{Q}$, and via the reciprocity map from class field theory the action of $\operatorname{Gal}\left(\mathbb{Q}^{a b} / \mathbb{Q}\right) \simeq \hat{\mathbb{Z}}^{\times}$on them is compatible under the isomorphism

$$
U \backslash \hat{B}^{\times} / B^{\times} \stackrel{\simeq}{\longrightarrow} \mathrm{n}(U) \backslash \hat{\mathbb{Q}}^{\times} / \mathbb{Q}^{\times}=\mathrm{n}(U) \backslash \hat{\mathbb{Z}}^{\times}
$$


induced by the reduced norm $\mathrm{n}$ on $\hat{B}^{\times}$(by strong approximation) with the natural action of $\hat{\mathbb{Z}}^{\times}$on $\mathrm{n}(U) \backslash \hat{\mathbb{Z}}^{\times}$.

From the very definition in (2), one can naturally define a group of automorphisms of $X_{U}$, which are often called modular. Namely, if $N(U)$ denotes the normalizer of $U$ in $\hat{B}^{\times}$, then left multiplication by an element $b \in N(U)$ induces an automorphism $\lambda(b): X_{U} \rightarrow X_{U}$, given on points by the rule

$$
\lambda(b):[g, f] \longmapsto[b g, f] .
$$

Here, $[g, f]$ denotes the point on $X_{U}$ corresponding to a pair $(g, f) \in$ $\hat{B}^{\times} \times \operatorname{Hom}\left(\mathbb{C}, \mathrm{M}_{2}(\mathbb{R})\right)$. It is immediate to check that $\lambda(b)$ defines the identity on $X_{U}$ if and only if $b \in U \mathbb{Q}^{\times}$. The group $\operatorname{Aut}^{\bmod }\left(X_{U}\right)$ of modular automorphisms on $X_{U}$ is then defined to be the group of all the automorphisms obtained in this way, so that

$$
\operatorname{Aut}^{\bmod }\left(X_{U}\right):=U \mathbb{Q}^{\times} \backslash N(U) .
$$

If $U=\hat{\mathcal{S}}^{\times}$is the group of units in the profinite completion of some order $\mathcal{S} \subseteq \mathcal{O}$, then we shall write $X_{\mathcal{S}}:=X_{\hat{\mathcal{S}} \times} / \mathbb{Q}$ for the Shimura curve associated with the order $\mathcal{S}$. In this case, the set of geometric connected components of $X_{\mathcal{S}}$ is identified with the class group $\operatorname{Pic}(\mathcal{S})$ of $\mathcal{S}$.

Remark 1.1. The most common setting in the literature is when $\mathcal{S}=$ $\mathcal{S}_{N^{+}}$is an Eichler order of level $N^{+}$in $\mathcal{O}$, where $N^{+} \geq 1$ is an integer prime to $N^{-}:=\Delta(B)$. In this case, the Shimura curve $X_{N^{+}, N^{-}}:=X_{\mathcal{S}} / \mathbb{Q}$ associated with $\mathcal{S}$ is not only connected but also geometrically connected, and its group Aut ${ }^{\bmod }\left(X_{N^{+}, N^{-}}\right)$of modular automorphisms is the group of Atkin-Lehner involutions, which are indexed by the positive divisors of $N^{+} N^{-}$. Further, $X_{N^{+}, N^{-}} / \mathbb{Q}$ is the coarse moduli space classifying abelian surfaces with quaternionic multiplication by $\mathcal{O}$ and $N^{+}$-level structure.

When $N^{-}=1$ (so that $B$ is the split quaternion algebra $\mathrm{M}_{2}(\mathbb{Q})$ ), a case which we exclude in this paper, the Shimura curve $X_{N^{+}, 1} / \mathbb{Q}$ is the affine modular curve $Y_{0}\left(N^{+}\right)$obtained as a quotient of the upper half plane by the congruence subgroup $\Gamma_{0}\left(N^{+}\right)$, whose compactification by adding finitely many cusps is the usual modular curve $X_{0}\left(N^{+}\right) / \mathbb{Q}$.

In this article, we will be working with Shimura curves associated with certain suborders of $\mathcal{O}$ which are not Eichler orders, but rather with more general orders that for example might have non-trivial level at primes dividing $\Delta(B)$. The special class of quaternion order we shall be dealing with is described in the next section. 
1.2. Choice of quaternion orders. Let $p$ be a rational prime and let $B_{p}$ be a quaternion algebra over $\mathbb{Q}_{p}$. The object of this section is introducing several families of local quaternion orders in $B_{p}$ which in turn will give rise to a fauna of Shimura curves that will serve as the appropriate host of the Heegner systems we aim to construct.

Assume first that $B_{p}=D_{p}$ is the unique (up to isomorphism) quaternion division algebra over $\mathbb{Q}_{p}$ and let $\mathcal{O}_{p}$ be the unique maximal order in $D_{p}$. If $L_{p}$ is a quadratic extension of $\mathbb{Q}_{p}$ and $\nu \geq 1$ is an integer, one can define the (local) quaternion order

$$
R_{\nu}\left(L_{p}\right)=\mathcal{O}_{L_{p}}+\varpi_{p}^{\nu-1} \mathcal{O}_{p}
$$

where $\mathcal{O}_{L_{p}}$ denotes the ring of integers of $L_{p}$ and $\varpi_{p}$ is a uniformizer element in $\mathcal{O}_{p}$. Such local orders are studied in detail by Hijikata, Pizer, and Shemanske in [HPS1]. Notice that $R_{1}\left(L_{p}\right)$ coincides with the maximal order $\mathcal{O}_{p}$, regardless of the choice of $L_{p}$. Further, if $L_{p}^{\prime}$ is another quadratic extension of $\mathbb{Q}_{p}$ with $L_{p} \simeq L_{p}^{\prime}$, then $R_{\nu}\left(L_{p}\right)$ and $R_{\nu}\left(L_{p}^{\prime}\right)$ are conjugated by an element in $D_{p}^{\times}$. For $\nu \geq 2$, the order $R_{\nu}\left(L_{p}\right)$ is characterized as the unique order in $D_{p}$ containing $\mathcal{O}_{L_{p}}$ and $\varpi_{p}^{\nu-1} \mathcal{O}_{p}$ but not containing $\varpi_{p}^{\nu-2} \mathcal{O}_{p}$.

Remark 1.2. If $p$ is odd and $L_{p}$ is the unique unramified quadratic extension, then $R_{2 r+1}\left(L_{p}\right)=R_{2 r+2}\left(L_{p}\right)$ for every $r \geq 0$, thus one can think of the orders $R_{\nu}\left(L_{p}\right)$ as being indexed by odd positive integers. These orders were studied in $[\mathbf{P i z 1}]$, where they are called orders of level $p^{2 r+1}$. When $p=2$ or $L_{p}$ is ramified, then $R_{\nu+1}\left(L_{p}\right) \subsetneq R_{\nu}\left(L_{p}\right)$ for every $\nu \geq 1$, and the order $R_{\nu}\left(L_{p}\right)$ depends in general on the choice of $L_{p}$. However, $R_{2}\left(L_{p}\right)$ is independent of $L_{p}$, and therefore one can speak of the unique order of level $p^{2}$ in $D_{p}(\mathrm{cf} .[\mathbf{P i z 2}])$.

Assume now that $B_{p}=\mathrm{M}_{2}\left(\mathbb{Q}_{p}\right)$ is the split quaternion algebra over $\mathbb{Q}_{p}$. In this algebra the order $\mathrm{M}_{2}\left(\mathbb{Z}_{p}\right)$ is maximal and it is the only one up to conjugation by elements in $\mathrm{GL}_{2}\left(\mathbb{Q}_{p}\right)$. Below we introduce, for each positive integer, two different $\mathrm{GL}_{2}\left(\mathbb{Z}_{p}\right)$-conjugacy classes of suborders in $\mathrm{M}_{2}\left(\mathbb{Z}_{p}\right)$, which therefore define two different $\mathrm{GL}_{2}\left(\mathbb{Q}_{p}\right)$-conjugacy classes of orders in $\mathrm{M}_{2}\left(\mathbb{Q}_{p}\right)$. Let $\nu \geq 1$ be an integer.

- The subring of $\mathrm{M}_{2}\left(\mathbb{Z}_{p}\right)$ consisting of those matrices $\left(\begin{array}{ll}a & b \\ c & d\end{array}\right)$ in $\mathrm{M}_{2}\left(\mathbb{Z}_{p}\right)$ such that $p^{\nu} \mid c$ is commonly referred to as the standard Eichler order of level $p^{\nu}$ in $\mathrm{M}_{2}\left(\mathbb{Z}_{p}\right)$. An Eichler order of level $p^{\nu}$ is then any order in $\mathrm{M}_{2}\left(\mathbb{Q}_{p}\right)$ which is conjugated to the standard one. We shall denote any of them by $R_{\nu}^{\text {Eic }}$, whenever only its conjugacy class is relevant in the discussion. 
- Let $\mathbb{Q}_{p^{2}}$ denote the unique unramified quadratic extension of $\mathbb{Q}_{p}$, and $\mathcal{O}=\mathbb{Z}_{p^{2}}$ be its valuation ring. Then $\mathcal{O} / p^{\nu} \mathcal{O}$ is a finite, free, commutative $\left(\mathbb{Z} / p^{\nu} \mathbb{Z}\right)$-algebra of rank 2 with unit discriminant. In particular, the choice of a basis for $\mathcal{O} / p^{\nu} \mathcal{O}$ gives an embedding of $\left(\mathcal{O} / p^{\nu} \mathcal{O}\right)^{\times}$into $\mathrm{GL}_{2}\left(\mathbb{Z} / p^{\nu} \mathbb{Z}\right)$. Its image $C_{\mathrm{ns}}\left(p^{\nu}\right)$ is then welldefined up to conjugation. The inverse image of $C_{\mathrm{ns}}\left(p^{\nu}\right) \cup\left\{\left(\begin{array}{ll}0 & 0 \\ 0 & 0\end{array}\right)\right\}$ under the reduction modulo $p^{\nu}$ map $\mathrm{M}_{2}\left(\mathbb{Z}_{p}\right) \rightarrow \mathrm{M}_{2}\left(\mathbb{Z} / p^{\nu} \mathbb{Z}\right)$ is an order of $\mathrm{M}_{2}\left(\mathbb{Z}_{p}\right)$, commonly referred to as a non-split Cartan order of level $p^{\nu}$. We shall denote any of the orders arising in this way simply by $R_{\nu}^{\mathrm{Car}}$, at any time that it is only the conjugacy class that matters in the discussion.

Now let $B / \mathbb{Q}$ be an indefinite quaternion algebra of discriminant $\Delta=$ $\Delta(B)$ as before.

Definition 1.3. Let $N_{\text {Eic }} \geq 1$ and $N_{\text {Car }} \geq 1$ be such that $\left(N_{\text {Eic }}, N_{\text {Car }}\right)=1$ and $\left(N_{\text {Eic }} \cdot N_{\text {Car }}, \Delta\right)=1$. For each prime $p \mid N_{\text {Eic }} \cdot N_{\text {Car }}$, set $\nu_{p}$ to be the $p$-adic valuation of $N_{\text {Eic }} \cdot N_{\text {Car }}$. For each prime $p \mid \Delta$, choose an integer $\nu_{p} \geq 1$ and a quadratic extension $L_{p}$ of $\mathbb{Q}_{p}$.

An order $\mathcal{R}$ in $B$ is said to be of type $T=\left(N_{\text {Eic }} ; N_{\text {Car }} ;\left\{\left(L_{p}, \nu_{p}\right)\right\}_{p \mid \Delta}\right)$ if the following conditions are satisfied:

(1) If $p \nmid N_{\text {Eic }} N_{\text {Car }} \Delta$, then $\mathcal{R} \otimes_{\mathbb{Z}} \mathbb{Z}_{p}$ is a maximal order in $B \otimes_{\mathbb{Q}} \mathbb{Q}_{p} \simeq$ $\mathrm{M}_{2}\left(\mathbb{Q}_{p}\right)$.

(2) If $p \mid N_{\text {Eic }}$, then $\mathcal{R} \otimes_{\mathbb{Z}} \mathbb{Z}_{p}$ is conjugate to $R_{\nu_{p}}^{\text {Eic }}$ in $B \otimes_{\mathbb{Q}} \mathbb{Q}_{p} \simeq \mathrm{M}_{2}\left(\mathbb{Q}_{p}\right)$.

(3) If $p \mid N_{\text {Car }}$, then $\mathcal{R} \otimes_{\mathbb{Z}} \mathbb{Z}_{p}$ is conjugate to $R_{\nu_{p}}^{\text {Car }}$ in $B \otimes_{\mathbb{Q}} \mathbb{Q}_{p} \simeq \mathrm{M}_{2}\left(\mathbb{Q}_{p}\right)$.

(4) For every $p \mid \Delta, \mathcal{R} \otimes_{\mathbb{Z}} \mathbb{Z}_{p} \simeq R_{\nu_{p}}\left(L_{p}\right)$ in $B \otimes_{\mathbb{Q}} \mathbb{Q}_{p} \simeq D_{p}$.

Remark 1.4. The way in which we have defined the above orders associated to the data $N_{\text {Eic }}, N_{\text {Car }}, \Delta$, and $\left\{\left(L_{p}, \nu_{p}\right)\right\}_{p \mid \Delta}$ is standard (cf. [Gro]), and the reason to consider such orders will become clearer later in the article. For a nice relation between the theory of local quaternion orders and the theory of ternary quadratic forms, the interested reader might consult $[\mathbf{L e m}]$.

Fix for the rest of this section an order $\mathcal{R}$ in $B$ of type $T=\left(N_{\text {Eic }} ; N_{\text {Car }}\right.$; $\left.\left\{\left(L_{p}, \nu_{p}\right)\right\}_{p \mid \Delta}\right)$ as in Definition 1.3. Define the level of $\mathcal{R}$ to be the integer $N_{\mathcal{R}}:=N_{\text {Eic }} \cdot N_{\text {Car }}^{2} \cdot N_{\Delta}$, where we put $N_{\Delta}:=\prod_{p \mid \Delta} p^{\nu_{p}}$. If $\nu_{p}=1$ for every $p \mid \Delta$, we will sometimes refer to $\mathcal{R}$ as a Cartan-Eichler order of type $\left(N_{\text {Eic }} ; N_{\text {Car }}\right)$ (and level $N_{\text {Eic }} \cdot N_{\text {Car }}^{2}$ ).

Associated with $\mathcal{R}$, we have the Shimura curve $X_{\mathcal{R}} / \mathbb{Q}$ defined as in the previous paragraph. The Shimura curve $X_{\mathcal{R}}$ is projective and smooth over $\mathbb{Q}$, but in general it is not geometrically connected. The reduced 
norm on $\mathcal{R}^{\times}$is locally surjective onto $\mathbb{Z}_{\ell}^{\times}$at every prime $\ell \nmid \Delta$ (both Eichler and Cartan orders in indefinite rational quaternion algebras have class number one), but however the reduced norm on the local orders $R_{\nu_{p}}\left(L_{p}\right)$ is not necessarily surjective onto $\mathbb{Z}_{p}^{\times}$when restricted to the invertible elements. Despite of this, it is easy to see that $\left[\mathbb{Z}_{p}^{\times}: \mathrm{n}\left(R_{\nu_{p}}\left(L_{p}\right)^{\times}\right)\right]$is either 1 or 2 . Thus if we set

$$
\mathcal{C}:=\left\{p \mid \Delta \text { prime: } \mathrm{n}\left(R_{\nu_{p}}\left(L_{p}\right)^{\times}\right) \neq \mathbb{Z}_{p}^{\times}\right\},
$$

then the number of connected components of $X_{\mathcal{R}} \times_{\mathbb{Q}} \overline{\mathbb{Q}}$ is $2^{|\mathcal{C}|}$. If $\Delta$ is odd, or if $\nu_{2} \leq 1$ in case that $\Delta$ is even, it follows from [HPS1, Theorem 3.11] that

$$
\mathcal{C}=\left\{p \mid \Delta \text { prime: } \nu_{p}>1, L_{p} \text { ramified }\right\} .
$$

The behaviour at $p=2$ is a bit more involved, but one still has a characterization of whether $\mathrm{n}\left(R_{\nu_{2}}\left(L_{2}\right)^{\times}\right)$has index 1 or 2 in $\mathbb{Z}_{2}^{\times}$(see [HPS1, Theorem $3.11,3$ ) and 4$)$ ] for details). Furthermore, if $\Delta$ is odd, the connected components of $X_{\mathcal{R}} \times \mathbb{Q} \overline{\mathbb{Q}}$ are defined over a polyquadratic extension: the number field obtained as the compositum of the quadratic extensions $\mathbb{Q}\left(\sqrt{p^{*}}\right)$ for $p \in \mathcal{C}$, where $p^{*}=\left(\frac{-1}{p}\right) p$.

Example 1.5. Suppose $\Delta=p q$ with $p$ and $q$ distinct odd primes, and let $L_{p}$ be a quadratic ramified extension of $\mathbb{Q}_{p}$. Consider an order $\mathcal{R}$ of type $\left(M ; 1 ;\left\{\left(L_{p}, 2\right),\left(L_{q}, 1\right)\right\}\right)$ and level $N=M p^{2} q$. As noticed in the above remark, this order does not depend on the choice of $L_{p}$. The Shimura curve $X_{\mathcal{R}} / \mathbb{Q}$ has two geometric connected components defined over the quadratic field $\mathbb{Q}\left(\sqrt{p^{*}}\right)$, and they are conjugated by the Galois action (in particular, they are isomorphic over $\mathbb{Q}\left(\sqrt{p^{*}}\right)$ ). There is a unique Eichler order $\mathcal{S}$ containing $\mathcal{R}$, and the morphism of Shimura curves $X_{\mathcal{R}} \rightarrow X_{\mathcal{S}}$ induced by the inclusion $\hat{\mathcal{R}}^{\times} \subseteq \hat{\mathcal{S}}^{\times}$is cyclic of degree $p+1$. Modular cusp forms in $S_{2}\left(\Gamma_{0}(N)\right)$ which are $N / M$-new and not principal series at $p$ lift via the Jacquet-Langlands correspondence to quaternionic modular forms on the Shimura curve $X_{\mathcal{R}}$ (see below).

\section{Modular forms and the Jacquet-Langlands correspondence}

We fix throughout this section an indefinite quaternion algebra $B$ of discriminant $\Delta$ and an order $\mathcal{R}$ of $B$ of type $T=\left(N_{\text {Eic }} ; N_{\text {Car }} ;\left\{\left(L_{p}, \nu_{p}\right)\right\}_{p \mid \Delta}\right)$ and level $N_{\mathcal{R}}=N_{\text {Eic }} N_{\text {Car }}^{2} N_{\Delta}$.

2.1. Cusp forms with respect to $\mathcal{R}$. We identify the Lie algebra of left invariant differential operators on $B_{\infty}^{\times}:=(B \otimes \mathbb{Q} \mathbb{R})^{\times} \simeq \mathrm{GL}_{2}(\mathbb{R})$ 
with $\mathrm{M}_{2}(\mathbb{C})$, and define the differential operators

$$
X_{\infty}=\left(\begin{array}{cc}
1 & \sqrt{-1} \\
\sqrt{-1} & -1
\end{array}\right), \bar{X}_{\infty}=\left(\begin{array}{cc}
1 & -\sqrt{-1} \\
-\sqrt{-1} & -1
\end{array}\right), W_{\infty}=\frac{1}{2}\left(\begin{array}{cc}
0 & -\sqrt{-1} \\
\sqrt{-1} & 0
\end{array}\right) .
$$

For each prime $p \mid \Delta$, write $\mathcal{R}_{p}^{1}=1+\varpi_{p}^{\nu_{p}-1} \mathcal{O}_{p}$ for the subgroup of units in $\mathcal{R}_{p}^{\times}$congruent to 1 modulo $\varpi_{p}^{\nu_{p}-1}$, where recall that $\mathcal{O}_{p}$ denotes the maximal order in the quaternion algebra $B_{p}$ and $\varpi_{p}$ is a uniformizer element. Then write $\hat{\mathcal{R}}^{1}$ for the subgroup of $\hat{\mathcal{R}}^{\times}$which is locally equal to $\hat{\mathcal{R}}^{\times}$everywhere away from $\Delta$, and equals $\mathcal{R}_{p}^{1}$ at each prime $p \mid \Delta$.

Definition 2.1. Let $k$ be an integer. A cusp form of weight $k$ with respect to $\hat{\mathcal{R}}^{1}$ is a function

$$
f:\left(B \otimes_{\mathbb{Q}} \mathbb{A}_{\mathbb{Q}}\right)^{\times}=\hat{B}^{\times} \times \mathrm{GL}_{2}(\mathbb{R}) \longrightarrow \mathbb{C}
$$

satisfying the following properties:

(1) if $g \in\left(B \otimes_{\mathbb{Q}} \mathbb{A}_{\mathbb{Q}}\right)^{\times}$, then the function $\mathrm{GL}_{2}(\mathbb{R}) \rightarrow \mathbb{C}$ given by $x \mapsto$ $f(x g)$ is of $C^{\infty}$-class and satisfies $W_{\infty} f=(k / 2) f, \bar{X}_{\infty} f=0$;

(2) for every $\gamma \in B^{\times}$and every $u \in \hat{\mathcal{R}}^{1} \times \mathbb{R}^{>0}, f(u g \gamma)=f(g)$.

The $\mathbb{C}$-vector space of all cusp forms of weight $k$ with respect to $\hat{\mathcal{R}}^{1}$ will be denoted $S_{k}\left(\mathcal{R}^{1}\right)$.

The product $\prod_{p \mid \Delta} B_{p}^{\times}$acts on the space $S_{k}\left(\mathcal{R}^{1}\right)$ by left translation, and through this action one can decompose $S_{k}\left(\mathcal{R}^{1}\right)$ into the direct sum of subspaces on which $\prod_{p \mid \Delta} B_{p}^{\times}$acts through some admissible representation, and for the purposes of this paper we are interested in those with trivial character. More precisely, suppose that for each $p \mid \Delta$ we are given an irreducible admissible representation $\rho_{p}$ of $B_{p}^{\times}$whose restriction to $\mathcal{R}_{p}^{\times}$is trivial, i.e. $\mathcal{R}_{p}^{\times} \subseteq \operatorname{ker}\left(\rho_{p}\right)$. Define $\rho:=\otimes_{p \mid \Delta} \rho_{p}$, regarded as a representation of $\prod_{p \mid \Delta} B_{p}^{\times}$. Since the representations $\rho_{p}$ are finitedimensional, the integer $d_{\rho}:=\operatorname{dim}(\rho)=\prod_{p \mid \Delta} \operatorname{dim}\left(\rho_{p}\right)$ is well-defined.

Definition 2.2. Let $k$ be an integer, and $\rho$ be a representation as above. A cusp form of weight $k$ with respect to $(\mathcal{R}, \rho)$ is a function

$$
f:\left(B \otimes_{\mathbb{Q}} \mathbb{A}_{\mathbb{Q}}\right)^{\times}=\hat{B}^{\times} \times \mathrm{GL}_{2}(\mathbb{R}) \longrightarrow \mathbb{C}^{d_{\rho}}
$$

satisfying the following conditions, for every $g \in\left(B \otimes_{\mathbb{Q}} \mathbb{A}_{\mathbb{Q}}\right)^{\times}$:

(1) for every $\gamma \in B^{\times}, f(g \gamma)=f(g)$;

(2) for every $b \in \prod_{p \mid \Delta} B_{p}^{\times}, f(b g)=\rho(b) f(g)$;

(3) for every prime $\ell \nmid \Delta$ and $u \in \mathcal{R}_{\ell}^{\times}, f(u g)=f(g)$;

(4) the function $\mathrm{GL}_{2}(\mathbb{R}) \rightarrow \mathbb{C}^{d_{\rho}}$ given by $x \mapsto f(x g)$ is of $C^{\infty}$-class and satisfies $W_{\infty} f=(k / 2) f, \bar{X}_{\infty} f=0$;

(5) for every $z \in \hat{\mathbb{Q}}^{\times} \times \mathbb{R}^{\times}, f(g z)=f(g)$. 
We write $S_{k}(\mathcal{R}, \rho)$ for the $\mathbb{C}$-vector space of cusp forms of weight $k$ with respect to $(\mathcal{R}, \rho)$.

The $\mathbb{C}$-vector spaces $S_{k}(\mathcal{R}, \rho)$ enjoy the following multiplicity one property:

Proposition 2.3 (cf. Proposition 2.14 in [Hid]). If two forms in $S_{k}(\mathcal{R}, \rho)$ are common eigenforms of the Hecke operators $T_{\ell}$ for all primes $\ell \nmid N$ with same eigenvalues, then they differ only by a constant factor.

The subspace of $S_{k}\left(\mathcal{R}^{1}\right)$ on which $\prod_{p \mid \Delta} B_{p}^{\times}$acts through an admissible representation $\rho$ as above is isomorphic to $S_{k}(\mathcal{R}, \rho)^{d_{\rho}}$, and we define $S_{k}(\mathcal{R})$ to be the subspace of $S_{k}\left(\mathcal{R}^{1}\right)$ given by

$$
S_{k}(\mathcal{R})=\bigoplus_{\rho} S_{k}(\mathcal{R}, \rho)^{d_{\rho}},
$$

where $\rho$ ranges over the representations $\rho=\otimes_{p \mid \Delta} \rho_{p}$ as above, satisfying $\mathcal{R}_{p}^{\times} \subseteq \operatorname{ker}\left(\rho_{p}\right)$.

Remark 2.4. The automorphic approach sketched before is related to the more classical point of view as follows. Let $h=h(\mathcal{R})$ denote the class number of $\mathcal{R}$ and choose elements $a_{i} \in \hat{B}^{\times}, i=1, \ldots, h$, such that

$$
\hat{B}^{\times}=\coprod_{i=1}^{h} \hat{\mathcal{R}}^{\times} a_{i} B^{\times} .
$$

Consider the discrete subgroups of $\mathrm{SL}_{2}(\mathbb{R})$ defined by

$$
\Gamma_{i}:=B_{+}^{\times} \cap a_{i}^{-1} \hat{\mathcal{R}}^{\times} a_{i} \quad(i=1, \ldots, h),
$$

where $B_{+}^{\times}$is the subgroup of units of positive reduced norm (we may write $B_{+}^{\times}=B^{\times} \cap \mathrm{GL}_{2}^{+}(\mathbb{R})$ using our identification of $B \otimes_{\mathbb{Q}} \mathbb{R}$ with $\mathrm{M}_{2}(\mathbb{R})$ ). If we denote by $S_{k}\left(\Gamma_{i}\right)$ the $\mathbb{C}$-vector space of cusp forms of weight $k$ with respect to the group $\Gamma_{i}$, then there is an isomorphism of complex vector spaces:

$$
\coprod_{i=1}^{h} S_{k}\left(\Gamma_{i}\right) \stackrel{\simeq}{\longrightarrow} S_{k}(\mathcal{R}) .
$$

2.2. Jacquet-Langlands. The space $S_{k}\left(\mathcal{R}^{1}\right)$ can be equipped with a standard action of Hecke operators and Atkin-Lehner involutions, described for example in $[\mathbf{H i d}]$. We have the following version of the Jacquet-Langlands correspondence: 
Theorem 2.5 (cf. Proposition 2.12 in [Hid]). There is a Hecke equivariant injection of $\mathbb{C}$-vector spaces

$$
S_{k}(\mathcal{R}, \rho) \longleftrightarrow S_{k}\left(\Gamma_{0}\left(N_{\text {Eic }} N_{\text {Car }}^{2} N_{\rho}\right)\right),
$$

where $N_{\rho}$ is the conductor of $\rho$.

Combining Theorem 2.5 with $(4)$ we can embed the space $S_{k}(\mathcal{R})$ into a space of classical modular cusp forms

$$
\mathrm{JL}: S_{k}(\mathcal{R}) \longleftrightarrow \bigoplus_{\rho} S_{k}\left(N_{\mathrm{Eic}} N_{\mathrm{Car}}^{2} N_{\rho}\right)^{d_{\rho}} .
$$

The multiplicities $d_{\rho}$ can be described explicitly: cf. [Car, $\left.\S 5\right]$.

Example 2.6. Suppose $p \mid \Delta$ is an odd prime. The quaternion algebra $B_{p}=B \otimes_{\mathbb{Q}} \mathbb{Q}_{p}$ is equipped with a natural decreasing filtration $\mathcal{O}_{p}^{\times}(i)$ defined by setting

$$
\mathcal{O}_{p}^{\times}(0)=\mathcal{O}_{p}^{\times} \quad \text { and } \quad \mathcal{O}_{p}^{\times}(i)=1+\varpi_{p}^{i} \mathcal{O}_{p},
$$

where $\mathcal{O}_{p}$ is the unique maximal order in $B_{p}$ and $\varpi_{p}$ is a local uniformizer. If $\rho_{p}$ is an admissible irreducible representation of $B_{p}^{\times}$, then its conductor is by definition $p^{n+1}$, where $n \geq 0$ is the smallest integer such that $\mathcal{O}_{p}^{\times}(n)$ lies in the kernel of $\rho_{p}$. In particular, observe that the conductor is at least $p$. Thus, if $p^{2} \nmid N_{\mathcal{R}}$ then $\mathcal{R}_{p}^{\times}=\mathcal{O}_{p}^{\times}$is precisely the group of units in the local maximal order at $p$, thus the admissible irreducible representations $\rho_{p}$ we are concerned with all have conductor $p$. If $p^{2} \mid N_{\mathcal{R}}$ and $p^{3} \nmid N_{\mathcal{R}}$, we have $\mathcal{O}_{p}^{\times}(1) \subseteq \mathcal{R}_{p}^{\times} \subseteq \mathcal{O}_{p}^{\times}$, and therefore the conductor of the admissible irreducible representations $\rho_{p}$ might be either $p$ or $p^{2}$. For each prime $p \mid \Delta$, the dimension of $\rho_{p}$ is determined by its minimal conductor, which by definition is the smallest conductor of the representations $\rho_{p} \otimes \chi$, as $\chi$ ranges over the characters of $\mathbb{Q}_{p}^{\times}$. By $[\mathbf{C a r}, \S 5]$, if the minimal conductor of $\rho_{p}$ is $p^{a}$, with $a \in\{1,2\}$, then $d_{\rho_{p}}=a$.

The above arguments give us a Hecke equivariant inclusion of $S_{k}(\mathcal{R})$ into a direct sum of spaces of classical modular cusp forms. In order to circumvent the problem of explicitly determining the multiplicities $d_{\rho}$, we use Proposition 2.8 below, which benefits from an explicit version of Eichler trace formula due to Hijikata, Pizer, and Shemanske.

For the reader's convenience, we recall the classification of JacquetLanglands lifts given in [HPS2], and from now on we focus on the weight 2 case. So let $f \in S_{2}\left(\Gamma_{0}\left(N_{f}\right)\right)$ be a weight 2 modular cusp form, and assume that $N_{f}=p^{s} M$ for some prime $p$ and integers $s, M \geq 1$, with $p \nmid M$. If $p=2$ and $s>3$, we assume that $s$ is odd. Suppose 
that $\phi$ is a Jacquet-Langlands lift of $f$ which is realized on the definite quaternion algebra $B^{(p)}$ of discriminant $p$. We want to determine the level of $\phi$, by which we mean the local $p$-type of the order $\mathcal{R}$ of $B^{(p)}$ used to define its level structure. Such local order is of the form $R_{n}(L)$, for some positive integer $n$ and quadratic extension $L / \mathbb{Q}_{p}$, and it is determined as follows:

(1) If $p$ is odd:

(a) $s$ odd: $L$ is unramified and $n=s$ [HPS2, Theorem 8.5].

(b) $s$ even: $L$ is ramified (any of the two ramified extensions) and $n=s$ [HPS2, Proposition 8.8, Case D].

(2) If $p=2$ :

(a) $s=1: L$ is the unramified quadratic extension of $\mathbb{Q}_{2}$ and $n=1$ [HPS2, Proposition 8.8, Case C].

(b) $s$ odd, $s \geq 3$ : $L$ is unramified and $n=s$ [HPS2, Theorem 8.5].

(c) $s=2: L=\mathbb{Q}_{2}(\sqrt{3})$ or $L=\mathbb{Q}_{2}(\sqrt{7})$ and $n=2[$ HPS2, Proposition 8.8, Case F, Eq. (8.17)].

Remark 2.7. If $p=2$ and $s \geq 4$ is even, [HPS2, Theorem 3.9] asserts that $f$ is a twist by a non-trivial character of conductor $2^{s / 2}$ of one of the cases considered above. However, as pointed out to us by the referee, there are known counterexamples which show that such statement is not true in general.

Proposition 2.8. Let $f \in S_{2}\left(\Gamma_{0}\left(N_{f}\right)\right)$ be a newform and fix a set $\Sigma$ of even cardinality consisting of primes $p \mid N_{f}$ such that the local admissible representation $\pi_{f, p}$ of $\mathrm{GL}_{2}\left(\mathbb{Q}_{\ell}\right)$ attached to $f$ is square-integrable. If $2 \in \Sigma$ and $2^{3} \mid N_{f}$, assume that $\operatorname{val}_{2}(N)$ is odd. Let $B / \mathbb{Q}$ be the indefinite quaternion algebra of discriminant $\Delta=\prod_{p \in \Sigma} p$, write $N_{\Delta}=$ $\prod_{p \in \Sigma} p^{\operatorname{val}_{p}\left(N_{f}\right)}$. Let $N_{\text {Eic }}, N_{\text {Car }}$ be positive integers so that $\left(N_{\text {Eic }}, N_{\text {Car }}\right)=$ 1 and $N_{\text {Eic }} N_{\text {Car }}^{2} N_{\Delta}=N_{f}$. Then, for any order $\mathcal{R}_{\min } \subset B$ of type $T_{\min }=\left(N_{\text {Eic }} ; N_{\text {Car }} ;\left\{\left(L_{p}, \operatorname{val}_{p}\left(N_{f}\right)\right)\right\}_{p \mid \Delta}\right)$, f lifts to a quaternionic modular form on $S_{2}\left(\mathcal{R}_{\min }\right)$ having the same Hecke eigenvalues for the Hecke operators $T_{\ell}$ at primes $\ell \nmid N_{f}$.

The subscript 'min' in $\mathcal{R}_{\text {min }}$ refers to the minimal level for primes dividing $\Delta$, determined by the classification explained above. For such primes, we note that if $\operatorname{val}_{p}\left(N_{f}\right)$ is odd then $L_{p}$ is unramified, and if $\operatorname{val}_{p}\left(N_{f}\right)$ is even then $L_{p}$ is ramified.

Proof: Since $\pi$ is square integrable at all primes in $\Sigma,[$ Gel, Theorem 10.2] implies the existence of an automorphic form $\pi^{\prime}$ on the algebraic group of invertible elements of the indefinite quaternion algebra $B$ 
as in the statement such that $\pi_{\ell}^{\prime} \simeq \pi_{\ell}$ for all primes $\ell$. To specify the order $\mathcal{R}$ we need to describe $\pi_{\ell}^{\prime}$ at every prime $\ell$. For primes $\ell \nmid N_{\text {Car }} \Delta$ the assertion is obvious. Fix a prime $p \mid \Delta$ and let $B^{(p)}$ be the definite quaternion algebra of discriminant $p$. Then Eichler's trace formula in [HPS2] shows the existence of an automorphic form $\pi^{(p)}$ for $B^{(p)}$ attached to a specific order $\mathcal{R}^{(p)}$ of type $\left(L_{p}, \operatorname{val}_{p}\left(N_{f}\right)\right)$ with $\pi_{p}^{\prime} \simeq \pi_{p}^{(p)}$ (we have sketched above the recipe for choosing $L_{p}$ ). Finally, for primes dividing $N_{\text {Car }}$ a similar argument works using this time the trace formula by Chen and Edixhoven (see [Edi, Theorem 1], [dSE, Theorem 2], and [Che, Section 6], or also [KP1, Theorem 1.11]; the proof in [Che] only works for $p \neq 2$, but one can check that it can be extended to the case $p=2$ ).

Example 2.9. Fix a primitive (in the sense of [Piz2, Definition 8.6]) new cuspidal eigenform $f$ of level $\Gamma_{0}\left(p^{2} M\right)$ as in the proposition with $p \nmid$ $M$ an odd prime. Let $B$ be the definite quaternion algebra of discriminant $p$ and $\mathcal{R}$ be an order of type $M p^{2}$ in $B$, accordingly with $[\mathbf{P i z 2}$, Definition 3.1] (which accordingly with the notation we adopted in the case of indefinite quaternion algebras, would correspond to orders of type $(M ; 1 ;(L, 2))$, for any choice of quadratic field $\left.L \subseteq B_{p}\right)$. It follows from [Piz2, Proposition 8.5 and Corollary 8.8] that $f$ appears twice in the space of quaternionic modular forms on $B$ of level $\mathcal{R}$ (which is the space spanned by Theta series, denoted $f_{i}$ and $g_{i}$ in loc. cit.). Since the multiplicity in Example 2.6 only depends on the behaviour of the local representation at $p$, we see that the subspace of new forms in $S_{2}(\mathcal{R})$ having the same system of Hecke eigenvalues as $f$ (at primes outside $N_{f}=p^{2} M$ ) is two-dimensional. So we have a "multiplicity 2 phenomenon" as expected from Example 2.6 and the Hecke-equivariant monomorphism JL in (5). Although this example involves modular forms on definite quaternion algebras, hence the global setting is of a different nature, we think it might be illustrative of an explicit example where the multiplicity is bigger than 1 .

2.3. Modular parametrizations. Let $J_{\mathcal{R}} / \mathbb{Q}$ denote the Jacobian variety of $X_{\mathcal{R}}$. It is a (principally polarized) abelian variety defined over $\mathbb{Q}$, of dimension equal to the genus of $X_{\mathcal{R}}$. Since $X_{\mathcal{R}}$ is not in general geometrically connected, it follows that $J_{\mathcal{R}}$ might be not absolutely simple. Recall the following:

Definition 2.10. An abelian variety $A / \mathbb{Q}$ is said to be modular if there exists a normalized newform $f=\sum_{n \geq 1} a_{n} q^{n}$ of weight 2 and level $\Gamma_{0}\left(N_{f}\right)$ 
for some $N_{f} \geq 1$ such that

$$
L(A, s)=\prod_{\sigma: F \hookrightarrow \overline{\mathbb{Q}}} L\left(f^{\sigma}, s\right),
$$

where $F$ stands for the number field generated by the Fourier coefficients of $f, \sigma$ ranges over the embeddings of $F$ into an algebraic closure of $\mathbb{Q}$, and $f^{\sigma}=\sum_{n \geq 1} \sigma\left(a_{n}\right) q^{n}$.

Proposition 2.11. Suppose that $A / \mathbb{Q}$ is a modular abelian variety associated with a modular form $f=\operatorname{JL}(\phi)$ for some $\phi \in S_{2}(\mathcal{R})$. Let $\mathbb{I}_{\phi} \subseteq \mathbb{T}$ be the kernel of the ring homomorphism $\mathbb{T} \rightarrow \mathbb{Z}$ determined by the system of Hecke eigenvalues of $\phi$. Then the quotient abelian variety $A_{\phi}:=J_{\mathcal{R}} / \mathbb{I}_{\phi} J_{\mathcal{R}}$ is isogenous to $A^{r}$ for some $r \geq 1$.

Proof: Let $\ell \nmid N_{f}$ be a prime and $\varrho: G_{\mathbb{Q}} \rightarrow \operatorname{Aut}\left(\operatorname{Ta}_{\ell}(A) \otimes \mathbb{Q}_{\ell}\right)$ be the 2-dimensional $\ell$-adic Galois representation arising from the natural action of $G_{\mathbb{Q}}$ on the $\ell$-adic Tate module $\operatorname{Ta}_{\ell}(A)$ of $A$. Similarly, let $\theta: G_{\mathbb{Q}} \rightarrow \operatorname{Aut}\left(\operatorname{Ta}_{\ell}\left(A_{\phi}\right) \otimes \mathbb{Q}_{\ell}\right)$ be the $\ell$-adic Galois representation attached to $A_{\phi}$. The Eichler-Shimura relations (proved in the required generality in [Nek2]) imply that $\theta(\sigma)$ is annihilated by the characteristic polynomial of $\varrho(\sigma)$ for every $\sigma \in G_{\mathbb{Q}}$. Then the Boston-Lenstra-Ribet Theorem $[\mathbf{B L R}]$ implies that $\operatorname{Ta}_{\ell}\left(A_{\phi}\right) \otimes \mathbb{Q}_{\ell}$ is isogenous to a direct sum of $r$ copies of $\operatorname{Ta}_{\ell}(A) \otimes \mathbb{Q}_{\ell}$ for some $r \geq 1$. Finally, Faltings' Isogeny Theorem implies that $A_{\phi}$ is isogenous to $r$ copies of $A$.

Example 2.12. Suppose that $\mathcal{R}$ is of type $\left(M ; 1 ;\left\{\left(L_{p}, 2\right),\left(L_{q}, 1\right)\right\}\right.$ and level $N:=N_{\mathcal{R}}=M p^{2} q$, with $p$ and $q$ distinct odd primes as in Example 1.5. Set $F=\mathbb{Q}\left(\sqrt{p^{*}}\right)$. Then

$$
J_{\mathcal{R}} \times_{\mathbb{Q}} F \sim J_{\mathcal{R}, 1} \times J_{\mathcal{R}, 2},
$$

where $J_{\mathcal{R}, i} / F$ is the Jacobian variety of $X_{\mathcal{R}, i}$. Let $\mathfrak{S}_{2}\left(\Gamma_{0}(N)\right)$ be the subspace of $S_{2}\left(\Gamma_{0}(N)\right)$ consisting of primitive newforms. By the same multiplicity 2 phenomenon observed in Example 2.9 (in a definite setting, but it is a local phenomenon, cf. also Example 2.6) there is a 2-to-1 Hecke-equivariant morphism of $\mathbb{C}$-vector spaces

$$
\mathfrak{S}_{2}(\mathcal{R}) \longrightarrow \mathfrak{S}_{2}\left(\Gamma_{0}(N)\right),
$$

where $\mathfrak{S}_{2}(\mathcal{R})$ is the subspace of modular forms $\phi \in S_{2}(\mathcal{R})$ such that $\operatorname{JL}(\phi) \in \mathfrak{S}_{2}\left(\Gamma_{0}(N)\right)$. By a slight abuse of notation we continue to denote by JL this morphism.

Fix $f \in \mathfrak{S}_{2}\left(\Gamma_{0}(N)\right)$ and assume that the Fourier coefficients of $f$ belongs to $\mathbb{Z}$. Then the abelian variety associated with $f$ is an elliptic curve $E$ of conductor $M p^{2} q$. Let $\phi \in \mathfrak{S}_{2}(\mathcal{R})$ be such that $\mathrm{JL}(\phi)=f$ 
(we have two linearly independent possible choices). The space $S_{2}(\mathcal{R})$ of weight 2 modular forms for $\mathcal{R}$ is identified with $H^{0}\left(X_{\mathcal{R}}, \Omega^{1}\right)$, which in turn is identified with the tangent space at the identity $T_{0}\left(J_{\mathcal{R}}\right)$ of $J_{\mathcal{R}}$. The subspace $\mathfrak{S}_{2}(\mathcal{R})$ corresponds then to a subspace of $H^{0}\left(X_{\mathcal{R}}, \Omega^{1}\right)$, and hence to the tangent space $T_{0}\left(\mathfrak{J}_{\mathcal{R}}\right)$ of an abelian subvariety $\mathfrak{J}_{\mathcal{R}}$ of $J_{\mathcal{R}}$. The space of modular forms $\mathfrak{S}_{2}(\mathcal{R})$ has rank 2 over the Hecke ring $\mathbb{T}$, and from this it follows that $T_{0}\left(\mathfrak{J}_{\mathcal{R}}\right) / T_{0}\left(\mathbb{I}_{\phi} \mathfrak{J}_{\mathcal{R}}\right)$ has dimension 2 over $\mathbb{Q}$. Hence $A_{\phi}$ is 2-dimensional, and therefore $A_{\phi} \sim E \times E$.

\section{Heegner points}

3.1. Optimal embeddings. As in previous sections, $B$ denotes an indefinite rational quaternion algebra of discriminant $\Delta=\Delta(B)$. We fix an order $\mathcal{R}$ and a quadratic field $K$. For each positive integer $c$ write $R_{c}$ for the (unique) order of conductor $c$ in $K, R_{1}$ being the full ring of integers of $K$.

Definition 3.1. Let $c$ be a positive integer. An embedding from $K$ to $B$, i.e. a $\mathbb{Q}$-algebra homomorphism $f: K \rightarrow B$, is said to be optimal with respect to $\mathcal{R} / R_{c}$ if the equality

$$
f(K) \cap \mathcal{R}=R_{c}
$$

holds. Since $f$ is determined by its restriction to $R_{c}$, we also speak of optimal embeddings of $R_{c}$ into $\mathcal{R}$.

Two optimal embeddings $f, f^{\prime}$ of $R_{c}$ into $\mathcal{R}$ will be considered to be equivalent if they are conjugate one to each other by an element in $\mathcal{R}^{\times}$. The set of $\mathcal{R}^{\times}$-conjugacy classes of optimal embeddings of $R_{c}$ into $\mathcal{R}$ will be denoted $\operatorname{Emb}^{\text {op }}\left(R_{c}, \mathcal{R}\right)$. We are interested in computing the integer

$$
v\left(R_{c}, \mathcal{R}\right)=\left|\mathrm{Emb}^{\text {op }}\left(R_{c}, \mathcal{R}\right)\right|,
$$

and in particular in knowing whether the set $\operatorname{Emb}^{\mathrm{op}}\left(R_{c}, \mathcal{R}\right)$ is empty or not.

Suppose now that $\mathcal{R}$ is of type $T=\left(N_{\text {Eic }} ; N_{\text {Car }} ;\left\{\left(L_{p}, \nu_{p}\right)\right\}_{p \mid \Delta}\right)$ and level $N_{\mathcal{R}}=N_{\text {Eic }} N_{\text {Car }}^{2} N_{\Delta}$. Recall that the class number $h(\mathcal{R})$ of the order $\mathcal{R}$ is $2^{|\mathcal{C}|}$, where $\mathcal{C}$ is the set introduced in (3). Although the class number of $\mathcal{R}$ is therefore not trivial in general, the lemma below asserts that the type number of $\mathcal{R}$ is always trivial, which amounts to saying that all orders in $B$ of the same type $T$ are conjugate one to each other.

Lemma 3.2. The type number of orders of a fixed type $T$ is 1 .

Proof: Fix a type $T=\left(N_{\text {Eic }} ; N_{\text {Car }} ;\left\{\left(L_{p}, \nu_{p}\right)\right\}_{p \mid \Delta}\right)$ as in Definition 1.3, and let $\mathcal{R}$ and $\mathcal{R}^{\prime}$ be two orders of type $T$ in $B$. First of all, notice that $\mathcal{R}$ 
(resp. $\mathcal{R}^{\prime}$ ) is a suborder of a unique Cartan-Eichler order $\mathcal{S}$ (resp. $\mathcal{S}^{\prime}$ ) of level $N_{\text {Eic }} N_{\text {Car }}^{2}$. Namely, the order which is locally equal to $\mathcal{R}$ (resp. $\mathcal{R}^{\prime}$ ) at every prime $p \nmid \Delta$ and locally maximal at primes $p \mid \Delta$, hence of type $\left(N_{\text {Eic }} ; N_{\text {Car }} ;\left\{\left(L_{p}, 1\right)\right\}_{p \mid \Delta}\right)$. Conversely, it is clear by construction that $\mathcal{R}$ (resp. $\mathcal{R}^{\prime}$ ) is the unique suborder of type $T$ of the Cartan-Eichler order $\mathcal{S}$ (resp. $\mathcal{S}^{\prime}$ ). The lemma now follows from the fact that the type number of Cartan-Eichler orders in $B$ is 1 , so that $\mathcal{S}$ and $\mathcal{S}^{\prime}$ are conjugate. By the above observation, this immediately implies that $\mathcal{R}$ and $\mathcal{R}^{\prime}$ are conjugate as well.

By virtue of the above lemma, the number $v\left(R_{c}, \mathcal{R}\right)$ can be expressed essentially as a product of local contributions that can be explicitly computed. Indeed, proceeding as in the proof of the 'trace formula' in [Vig, Chapter III, 5.C] (cf. especially Theorems 5.11 and 5.11 bis, or [Brz1]) for Eichler orders, we have that

$$
v\left(R_{c}, \mathcal{R}\right)=\frac{h\left(R_{c}\right)}{h(\mathcal{R})} \prod_{\ell} v_{\ell}\left(R_{c}, \mathcal{R}\right)
$$

where $h\left(R_{c}\right)$ (resp. $h(\mathcal{R})$ ) is the class number of the quadratic order $R_{c}$ (resp. of $\mathcal{R}$ ), the product ranges over all rational primes and, for each $\ell$, $v_{\ell}\left(R_{c}, \mathcal{R}\right)$ denotes the number of local optimal embeddings of $R_{c} \otimes_{\mathbb{Z}} \mathbb{Z}_{\ell}$ into $\mathcal{R} \otimes_{\mathbb{Z}} \mathbb{Z}_{\ell}$ modulo conjugation by $\left(\mathcal{R} \otimes_{\mathbb{Z}} \mathbb{Z}_{\ell}\right)^{\times}$. These local contributions are 1 for every prime $\ell \nmid N$. The number of local optimal embeddings is determined in $\S 3.2$ below. Here we give the following:

Example 3.3. Assume that $N_{\text {Car }}=1, \Delta$ is odd, and $\nu_{p} \leq 2$ for all $p \mid \Delta$. Then

$$
v\left(R_{c}, \mathcal{R}\right)=\frac{h\left(R_{c}\right)}{h(\mathcal{R})} \prod_{\ell \mid N_{\text {Eic }}}\left(1+\left\{\frac{R_{c}}{\ell}\right\}\right) \prod_{\substack{q \mid \Delta \\ \nu(q)=1}}\left(1-\left\{\frac{R_{c}}{\ell}\right\}\right) \prod_{\substack{p \mid \Delta \\ \nu_{p}=2}} v_{p}\left(R_{c}, \mathcal{R}\right)
$$

where for primes $p \mid \Delta$ with $\nu_{p}=2$,

$$
v_{p}\left(R_{c}, \mathcal{R}\right)= \begin{cases}2 & \text { if } p \| c \text { and } p \text { is inert in } K, \\ p+1 & \text { if } p \nmid c \text { and } p \text { ramifies in } K, \\ 0 & \text { otherwise. }\end{cases}
$$

Here $\left\{\frac{R}{\ell}\right\}$ denotes the usual Eichler symbol attached to a quadratic order $R$ and a prime number $\ell$. 
3.2. Local optimal embeddings. For the reader's convenience, we reproduce in this subsection the criteria for the existence of local optimal embeddings of orders in quadratic fields into quaternion orders in the Eichler, Cartan, and division cases.

3.2.1. Eichler case. Let $p$ be a prime, $K / \mathbb{Q}_{p}$ be a quadratic separable algebra, and $\mathcal{O}_{m} \subseteq K$ be the order in $K$ of conductor $p^{m}$. Let also $\mathrm{M}_{2}\left(\mathbb{Q}_{p}\right)$ be the split quaternion algebra over $\mathbb{Q}_{p}$ and $R_{n}^{\text {Eic }}$ be the standard Eichler order of level $p^{n}$ in $\mathrm{M}_{2}\left(\mathbb{Q}_{p}\right)$. Write $h(m, n)$ for the number of (equivalence classes of) optimal embeddings of $\mathcal{O}_{m}$ into $R_{n}^{\text {Eic }}$.

Suppose first that $K=\mathbb{Q}_{p} \oplus \mathbb{Q}_{p}$ is the split quadratic $\mathbb{Q}_{p}$-algebra. Then $m$ is the smallest positive integer such that $\mathcal{O}_{m} / p^{m} \mathcal{O}_{m} \simeq \mathbb{Z} / p^{m} \mathbb{Z}$. In this case, the embedding $(a, b) \mapsto\left(\begin{array}{cc}a & 0 \\ p^{n-m}(a-b) & b\end{array}\right)$ from $K$ into $\mathrm{M}_{2}\left(\mathbb{Q}_{p}\right)$ defines an optimal embedding from $\mathcal{O}_{m}$ into $R_{n}^{\text {Eic }}$. For later reference, we state the following lemma.

Lemma 3.4. If $K$ is the split quadratic $\mathbb{Q}_{p}$-algebra, then $\mathcal{O}_{m}$ can be optimally embedded in $R_{n}^{\text {Eic }}$ for every $m \geq 0$. That is, $h(m, n) \neq 0$ for every $m \geq 0$.

Next we assume that $K / \mathbb{Q}_{p}$ is a quadratic field extension with valuation ring $\mathcal{O}$, and again for each $m \geq 1$ let $\mathcal{O}_{m}$ be the order of conductor $p^{m}$ in $K$. Recall that the Eichler symbol is defined as follows:

$$
\left\{\frac{\mathcal{O}_{m}}{p}\right\}= \begin{cases}-1 & \text { if } m=0 \text { and } K / \mathbb{Q}_{p} \text { is unramified } \\ 0 & \text { if } m=0 \text { and } K / \mathbb{Q}_{p} \text { is ramified } \\ 1 & \text { if } m \geq 1\end{cases}
$$

It is well known $([\mathbf{H i j}],[\mathbf{V i g}])$ that if $n=0$ then $h(m, n)=1$, and for $n=1$ one has $h(m, n)=1+\left\{\frac{\mathcal{O}_{m}}{p}\right\}$. Thus, in particular, every quadratic order $\mathcal{O}_{m}$ can be optimally embedded in the maximal Eichler order unless $m=0$ and $K / \mathbb{Q}_{p}$ is unramified, the only case when $h(m, 1)=0$. More generally (see [Brz2, Corollary 1.6]):

Lemma 3.5. (1) If $K / \mathbb{Q}_{p}$ is unramified, then $h(m, n) \neq 0$ if and only if $m \geq n / 2$.

(2) If $K / \mathbb{Q}_{p}$ is ramified, then $h(m, n) \neq 0$ if and only if $m \geq(n-1) / 2$.

3.2.2. Cartan case. Let $p$ be a prime, $K=\mathbb{Q}_{p^{2}}$ be the unramified quadratic extension of $\mathbb{Q}_{p}$, and $\mathcal{O}=\mathbb{Z}_{p^{2}}$ be its valuation ring. As above, for $m \geq 1$ write $\mathcal{O}_{m}$ for the order of conductor $p^{m}$ in $K$. From the very definition of non-split Cartan orders, we have the following lemma, which we state for later reference: 
Lemma 3.6. Let $R_{n}^{\text {Car }}$ be a non-split Cartan order of level $p^{n}$ in $\mathrm{M}_{2}\left(\mathbb{Q}_{p}\right)$. Then $\mathcal{O}$ can be optimally embedded in $R_{n}^{\text {Car }}$. For $m>1$, the order $\mathcal{O}_{m}$ does embed in $R_{n}^{\text {Car }}$, but not optimally.

3.2.3. Division case ([HPS1]). Let $p$ be a prime, and $D_{p}$ be the unique division quaternion algebra over $\mathbb{Q}_{p}$. As above, write $R_{n}(L)$ for the local order in $D_{p}$ associated to the choice of an integer $n \geq 1$ and a quadratic extension $L / \mathbb{Q}_{p}$. Let $K / \mathbb{Q}_{p}$ be a quadratic field extension, and $\mathcal{O}_{m}$ denote the order of conductor $p^{m}$ in $K$ as before. Recall that $h(m, n, L)$ denotes the number of equivalence classes of optimal embeddings of $\mathcal{O}_{m}$ into $R_{n}(L)$.

It might be useful first to recall the notation used in [HPS1] for the symbols $t(L)$ and $\mu\left(L, L^{\prime}\right)$. For any quadratic field extension $L / \mathbb{Q}_{p}$ :

- $t(L)=-1$ means $L$ unramified;

- $t(L)=0$ means $L$ ramified and $p \neq 2$;

- $t(L)=1$ means $p=2$ and $L=\mathbb{Q}_{p}(\sqrt{3})$, or $L=\mathbb{Q}_{p}(\sqrt{7})$;

- $t(L)=2$ means $p=2$ and $L=\mathbb{Q}_{p}(\sqrt{2}), L=\mathbb{Q}_{p}(\sqrt{6}), L=\mathbb{Q}_{p}(\sqrt{10})$, or $L=\mathbb{Q}_{p}(\sqrt{14})$.

And for any pair of quadratic field extensions $\left(L, L^{\prime}\right)$ of $\mathbb{Q}_{p}$ having discriminants $\Delta(L)$ and $\Delta\left(L^{\prime}\right)$ we have:

- $\mu\left(L, L^{\prime}\right)=\mu\left(L^{\prime}, L\right)$ (Theorem 3.10 A (iii) of [HPS1]);

- if $\Delta(L)=\Delta\left(L^{\prime}\right)$ (which is the case if $L \simeq L^{\prime}$ ), then $\mu\left(L, L^{\prime}\right)=\infty$;

- if $t(L)=-1$ and $\Delta(L) \neq \Delta\left(L^{\prime}\right)$, then $\mu\left(L, L^{\prime}\right)=1$;

- if $t(L)=0, t\left(L^{\prime}\right)=0$, and $\Delta(L) \neq \Delta\left(L^{\prime}\right)$, then $\mu\left(L, L^{\prime}\right)=2$;

- if $t(L)=1, t\left(L^{\prime}\right)=1$, and $\Delta(L) \neq \Delta\left(L^{\prime}\right)$, then $\mu\left(L, L^{\prime}\right)=3$;

- if $t(L)=1, t\left(L^{\prime}\right)=2$, then $\mu\left(L, L^{\prime}\right)=3$;

- if $t(L)=2, t\left(L^{\prime}\right)=2$, and $\Delta(L) \neq \Delta\left(L^{\prime}\right)$, then $\mu\left(L, L^{\prime}\right)=5$.

The criteria for the existence of optimal embeddings then reads as follows:

(1) $p$ odd:

(a) $n=2 \varrho+1$ odd, $K$ unramified, $L$ unramified: $h(m, n, L) \neq 0$ if and only if $m \leq \varrho$. In particular, if $R_{n}(L)$ is maximal and $\mathcal{O}_{m}$ is not maximal (i.e. $m>0$ and $n=0$ ), then $h(m, n, L)=0$.

(b) $n=2 \varrho+1$ odd, $K$ ramified, $L$ unramified: $h(m, n, L) \neq 0$ if and only if $m=\varrho$. In particular, if $R_{n}(L)$ is maximal and $\mathcal{O}_{m}$ is not maximal (i.e. $m>0$ and $\varrho=0$ ), then $h(m, n, L)=0$.

(c) $n=2 \varrho$ even, $K$ unramified, $L$ ramified: $h(m, n, L) \neq 0$ if and only if $m=\varrho$. 
(d) $n=2 \varrho$ even, $K$ ramified, $L$ ramified and $K \not L L: h(m, n, L) \neq$ 0 if and only if $m=\varrho-1$.

(e) $n=2 \varrho$ even, $K$ ramified, $L$ ramified and $K \simeq L: h(m, n, L) \neq$ 0 if and only if $m \leq \varrho-1$.

(2) $p=2$ :

(a) $n=1, K$ ramified or unramified, $L$ unramified: $h(m, n, L) \neq 0$ if and only if $m=0$.

(b) $n=2 \varrho, K$ unramified, $L=\mathbb{Q}_{2}(\sqrt{3})$ or $L=\mathbb{Q}_{2}(\sqrt{7})$; this is the case of $t(L)=1, t(K)=-1$ and therefore $\mu(L, K)=1$ : $h(m, n, L) \neq 0$ if and only if $m=\rho$.

(c) $n=2 \varrho, K=\mathbb{Q}_{2}(\sqrt{3})$ or $K=\mathbb{Q}_{2}(\sqrt{7}), L=\mathbb{Q}_{2}(\sqrt{3})$ or $L=$ $\mathbb{Q}_{2}(\sqrt{7})$ and $K \not 4 L$; this is the case of $t(L)=1, t(K)=1$ and $\Delta(L) \neq \Delta(K)$ and therefore $\mu(L, K)=3: h(m, n, L) \neq 0$ if and only if $m=\rho-1$.

(d) $n=2 \varrho, K=\mathbb{Q}_{2}(\sqrt{3})$ or $K=\mathbb{Q}_{2}(\sqrt{7})$ and $K \simeq L$; this is the case of $t(L)=1, t(K)=1$ and $\Delta(L)=\Delta(K)$ and therefore $\mu(L, K)=\infty: h(m, n, L) \neq 0$ if and only if $m \leq \rho-1$.

(e) $n=2 \varrho, K=\mathbb{Q}_{p}(\sqrt{2}), K=\mathbb{Q}_{p}(\sqrt{6}), K=\mathbb{Q}_{p}(\sqrt{10})$ or $K=$ $\mathbb{Q}_{p}(\sqrt{14}), L=\mathbb{Q}_{2}(\sqrt{3})$ or $L=\mathbb{Q}_{2}(\sqrt{7})$; this is the case of $t(L)=$ $1, t(K)=2$ and therefore $\mu(L, K)=3: h(m, n, L) \neq 0$ if and only if $m=\rho-1$.

(f) $n=2 \varrho+1$ odd, $n \geq 3, K$ unramified and $L$ unramified: $h(m, n, L) \neq 0$ if and only if $m \leq \varrho$.

(g) $n=2 \varrho+1$ odd, $n \geq 3, K$ ramified and $L$ unramified: $h(m, n, L) \neq$ 0 if and only if $m=\varrho$.

(h) $n=2 \varrho, K$ unramified and $L$ ramified. Then $t(L)=1$ or 2 and $\mu(L, K)=1: h(m, n, L) \neq 0$ if and only if $m=\varrho$.

(i) $n=2 \varrho, K=\mathbb{Q}_{2}(\sqrt{3})$ or $K=\mathbb{Q}_{p}(\sqrt{7}), L=\mathbb{Q}_{2}(\sqrt{2}), L=\mathbb{Q}_{2}(\sqrt{6})$, $L=\mathbb{Q}_{2}(\sqrt{10})$ or $L=\mathbb{Q}_{2}(\sqrt{14})$. Then $t(L)=2, t(K)=1$, $\mu(L, K)=3: h(m, n, L) \neq 0$ if and only if $m=\varrho-1$.

(j) $n=2 \varrho, K=\mathbb{Q}_{2}(\sqrt{2}), K=\mathbb{Q}_{2}(\sqrt{6}), K=\mathbb{Q}_{2}(\sqrt{10})$ or $K=$ $\mathbb{Q}_{2}(\sqrt{14}), L=\mathbb{Q}_{2}(\sqrt{2}), L=\mathbb{Q}_{2}(\sqrt{6}), L=\mathbb{Q}_{2}(\sqrt{10})$ or $L=$ $\mathbb{Q}_{2}(\sqrt{14})$ and $K \not z L$. Then $t(L)=2, t(K)=2, \mu(L, K)=5$ : $h(m, n, L) \neq 0$ if and only if $m=\varrho-1$ or $m=\varrho-2$.

(k) $n=2 \varrho, K=\mathbb{Q}_{2}(\sqrt{2}), K=\mathbb{Q}_{2}(\sqrt{6}), K=\mathbb{Q}_{2}(\sqrt{10})$ or $K=$ $\mathbb{Q}_{2}(\sqrt{14})$, and $K \simeq L$. Then $t(L)=2, t(K)=2, \mu(L, K)=\infty$ : $h(m, n, L) \neq 0$ if and only if $m \leq \varrho-1$.

3.3. Heegner points. Let $U$ be any open compact subgroup of $\hat{B}^{\times}$, and assume that $K$ is an imaginary quadratic field. There is a natural 
map

$$
\hat{B}^{\times} \times \operatorname{Hom}(K, B) \longrightarrow\left(U \backslash \hat{B}^{\times} \times \operatorname{Hom}\left(\mathbb{C}, \mathrm{M}_{2}(\mathbb{R})\right)\right) / B^{\times}=X_{U}(\mathbb{C})
$$

obtained by extending scalars (i.e., tensoring with $\mathbb{R}$ ). Notice that the left-hand side can certainly be the empty set, as $\operatorname{Hom}(K, B)$ is empty if $K$ does not embed into $B$. We shall assume that this is not the case in the discussion below. If $(g, f) \in \hat{B}^{\times} \times \operatorname{Hom}(K, B)$, write $[g, f]$ for its image in $X_{\mathcal{R}}(\mathbb{C})$. Points in the image of this map are called Heegner points; the set of such Heegner points is denoted $\operatorname{Heeg}(U, K)$.

For each positive integer $c$, continue to denote by $R_{c}$ the order of conductor $c$ in $K$ and let $\mathcal{R}$ be an order of $B$.

Definition 3.7. A point $x \in X_{\mathcal{R}}$ is called a Heegner point of conductor $c$ associated to $K$ if $x=[g, f]$ for some pair $(g, f) \in \hat{B}^{\times} \times \operatorname{Hom}(K, B)$ such that

$$
f(K) \cap g^{-1} \hat{\mathcal{R}} g=f\left(R_{c}\right) .
$$

This last condition means that $f$ is an optimal embedding of $R_{c}$ into the order $g^{-1} \hat{\mathcal{R}} g \cap B$. We shall denote by $\operatorname{Heeg}(\mathcal{R}, K, c)$ the set of Heegner points of conductor $c$ associated to $K$ in $X_{\mathcal{R}}$.

Recall that the set of geometrically connected components of the Shimura curve $X_{\mathcal{R}}$ is in bijection with $\hat{\mathcal{R}}^{\times} \backslash \hat{B}^{\times} / B^{\times}$, and hence with the class group $\operatorname{Pic}(\mathcal{R})$ of $\mathcal{R}$. In particular, the number of geometric connected components coincides with the class number $h(\mathcal{R})$. Fix representatives $I_{j}$ for the distinct $h(\mathcal{R})$ ideal classes in $\operatorname{Pic}(\mathcal{R})$, and let $a_{j} \in \hat{B}^{\times}$be the corresponding representatives in $\hat{\mathcal{R}}^{\times} \backslash \hat{B}^{\times} / B^{\times}$. It is then clear that every Heegner point in $\operatorname{Heeg}(\mathcal{R}, K, c)$ can be represented by a pair of the form $\left(a_{j}, f\right)$, for a unique $j \in\{1, \ldots, h(\mathcal{R})\}$ and some optimal embedding $f$ from $R_{c}$ into the order $a_{j}^{-1} \hat{\mathcal{R}} a_{j} \cap B$. Further, two pairs $\left(a_{j}, f\right)$ and $\left(a_{j}, g\right)$ represent the same Heegner point if and only if the embeddings $f$ and $g$ are $\mathcal{R}^{\times}$-conjugate. Therefore, we have the following identity relating Heegner points on $X_{\mathcal{R}}$ attached to $R_{c}$ and optimal embeddings of $R_{c}$ into $\mathcal{R}$ :

$$
|\operatorname{Heeg}(\mathcal{R}, K, c)|=h(\mathcal{R})\left|\operatorname{Emb}^{\text {op }}\left(R_{c}, \mathcal{R}\right)\right|=h(\mathcal{R}) v\left(R_{c}, \mathcal{R}\right),
$$

thus applying (6) we find:

Proposition 3.8. The number of Heegner points on $X_{\mathcal{R}}$ attached to $R_{c}$ is $h\left(R_{c}\right) \prod_{\ell} v_{\ell}\left(R_{c}, \mathcal{R}\right)$. 
3.4. Galois action and fields of rationality. Keep the same notations as above, and assume that $R_{c}$ embeds optimally in $\mathcal{R}$, so that Heegner points with respect to $R_{c}$ do exist on $X_{\mathcal{R}}$. The reciprocity law, cf. [Del1, 3.9], [Mil1, II.5.1] (with a sign corrected [Mil2, 1.10]), asserts that $\operatorname{CM}(\mathcal{R}, K, c) \subseteq X_{\mathcal{R}}\left(K^{a b}\right)$, where as usual $K^{a b}$ denotes the maximal abelian extension of $K$, and further that the Galois action of $\operatorname{Gal}\left(K^{a b} / K\right)$ on $\operatorname{CM}(\mathcal{R}, K, c)$ is described by

$$
\operatorname{rec}_{K}(a)[g, f]=[\hat{f}(a) g, f], \quad\left(a \in \hat{K}^{\times}\right) .
$$

Here, $\operatorname{rec}_{K}: \hat{K}^{\times} \rightarrow \operatorname{Gal}\left(K^{a b} / K\right)$ is the reciprocity map from class field theory. Then, for an arbitrary $a \in \hat{K}^{\times}$and every Heegner point $[g, f]$ we have

$$
\begin{array}{r}
\operatorname{rec}_{K}(a)[g, f]=[g, f] \Longleftrightarrow \text { there exist } b \in B^{\times}, u \in \hat{\mathcal{R}}^{\times} \\
\text {such that }(\hat{f}(a) g, f)=\left(u g b, b^{-1} f b\right) .
\end{array}
$$

It is easy to show that if $f: K \rightarrow B$ is an embedding and $b \in B^{\times}$, then the equality $f=b^{-1} f b$ holds if and only if $b=f(\lambda)$ for some $\lambda \in K^{\times}$. Thus we deduce that

$$
\begin{aligned}
\operatorname{rec}_{K}(a)[g, f]=[g, f] & \Longleftrightarrow \text { there exist } \lambda \in K^{\times}, u \in \hat{\mathcal{R}}^{\times} \\
& \text {such that } \hat{f}(a)=g^{-1} u g f(\lambda) \\
& \Longleftrightarrow a \in \hat{f}^{-1}\left(g^{-1} \hat{\mathcal{R}}^{\times} g\right) K^{\times}=\hat{R}_{c}^{\times} K^{\times} .
\end{aligned}
$$

By class field theory, $\operatorname{rec}_{K}$ induces an isomorphism

$$
\hat{K}^{\times} / \hat{R}_{c}^{\times} K^{\times}=\operatorname{Pic}\left(R_{c}\right) \stackrel{\simeq}{\longrightarrow} \operatorname{Gal}\left(H_{c} / K\right),
$$

where $H_{c}$ is the ring class field of conductor $c$. Hence we have proved:

Proposition 3.9. With notations as above, $\operatorname{Heeg}(\mathcal{R}, K, c) \subseteq X_{\mathcal{R}}\left(H_{c}\right)$, and the action of $\operatorname{Gal}\left(H_{c} / K\right)$ on the set of Heegner points $\operatorname{Heeg}(\mathcal{R}, K, c)$ is described by the rule in (7).

\section{Applications}

4.1. Gross-Zagier formula. We briefly review the general form of Gross-Zagier formula in [YZZ] for modular abelian varieties. Let $B / \mathbb{Q}$ be an indefinite quaternion algebra of discriminant $\Delta$. If $U_{1} \subseteq U_{2}$ are open compact subgroups of $\hat{B}^{\times}$, then we have a canonical projection map $\pi_{U_{1}, U_{2}}: X_{U_{1}} \rightarrow X_{U_{2}}$, and one may consider the projective limit

$$
X=\lim _{U} X_{U}
$$

and let $J:=\operatorname{Jac}(X)$ denote the Jacobian variety of $X$. 
Definition 4.1. A simple abelian variety $A / \mathbb{Q}$ is said to be uniformized by $X$ if there exists a surjective morphism $J \rightarrow A$ defined over $\mathbb{Q}$.

Let $A / \mathbb{Q}$ be a simple abelian variety uniformized by $X$ and fix $U$ such that there is a surjective morphism $J_{U}:=\operatorname{Jac}\left(X_{U}\right) \rightarrow A$ defined over $\mathbb{Q}$. Let $\xi_{U}$ be the normalized Hodge class in $X_{U}$ and define

$$
\pi_{A}:=\underset{U}{\lim } \operatorname{Hom}_{\xi_{U}}^{0}\left(X_{U}, A\right),
$$

where $\operatorname{Hom}_{\xi_{U}}^{0}\left(X_{U}, A\right)$ denotes morphisms of $\operatorname{Hom}\left(X_{U}, A\right) \otimes_{\mathbb{Z}} \mathbb{Q}$ defined by using the Hodge class $\xi_{U}$ as a base point. Since, by the universal property of Jacobians, every morphism $X_{U} \rightarrow A$ factors through $J_{U}$, we also have

$$
\pi_{A}:=\underset{U}{\lim } \operatorname{Hom}_{\xi_{U}}^{0}\left(J_{U}, A\right)
$$

where $\operatorname{Hom}_{\xi_{U}}^{0}\left(J_{U}, A\right):=\operatorname{Hom}\left(J_{U}, A\right) \otimes_{\mathbb{Z}} \mathbb{Q}$. For any $\varphi \in \pi_{A}$ and any point $P \in X_{U}(H)$, where $H / \mathbb{Q}$ is a field extension, we then see that $P(\varphi):=\varphi(P) \in A(H)$.

Let $K / \mathbb{Q}$ be an imaginary quadratic field and assume there exists an embedding $\psi: K \hookrightarrow B$; this is equivalent to say that all primes dividing $\Delta$ are inert or ramified in $K$. Define $X^{K^{\times}}$to be the subscheme of $X$, defined over $\mathbb{Q}$, consisting of fixed points under the canonical action by left translation of $\hat{\psi}: \hat{K}^{\times} \hookrightarrow \hat{B}^{\times}$. The subscheme $X^{K^{\times}}$is independent up to translation of the choice of $\psi$. We will often omit the reference to $\psi$, viewing $K$ simply as a subfield of $B$. Recall that the theory of complex multiplication shows that every point in $X^{K^{\times}}(\overline{\mathbb{Q}})$ is defined over $K^{\mathrm{ab}}$, the maximal abelian extension of $K$, and that the Galois action is given by left translation under the reciprocity map. Fix a point $P \in X^{K^{\times}}\left(K^{\mathrm{ab}}\right)$. This amounts to choose a point $P_{U}$ for all open compact subgroups $U$, satisfying the condition that $\pi_{U_{1}, U_{2}}\left(P_{U_{1}}\right)=P_{U_{2}}$.

Let $d \tau$ denote the Haar measure of $\operatorname{Gal}\left(K^{\mathrm{ab}} / K\right)$ of total mass equal to 1 and fix a finite order character $\chi: \operatorname{Gal}\left(K^{\mathrm{ab}} / K\right) \rightarrow F_{\chi}^{\times}$, where $F_{\chi}=$ $\mathbb{Q}(\chi)$ is the finite field extension of $\mathbb{Q}$ generated by the values of $\chi$. Define

$$
P_{\chi}(\varphi):=\int_{\operatorname{Gal}\left(K^{\mathrm{ab} / K}\right)} \varphi\left(P^{\tau}\right) \otimes \chi(\tau) d \tau .
$$

This is an element in $A\left(K^{\mathrm{ab}}\right) \otimes_{M} F_{\chi}$, where $M=\operatorname{End}_{\mathbb{Q}}^{0}(A):=\operatorname{End}_{\mathbb{Q}}(A) \otimes_{\mathbb{Z}}$ $\mathbb{Q}$. This element can be essentially written as a finite sum: suppose that $P=\left(P_{U}\right)_{U}$, and each $P_{U}$ is defined over the abelian extension $H_{U}$ of $K$. Suppose that $\chi$ factors through $\operatorname{Gal}\left(H_{U} / K\right)$ for some $U$. Then 
the $F_{\chi}$-subspace of $A\left(H_{U}\right) \otimes F_{\chi}$ spanned by $P_{\chi}(\varphi)$ and

$$
\sum_{\sigma \in \operatorname{Gal}\left(H_{U} / K\right)} \varphi(P)^{\sigma} \otimes \chi(\sigma)
$$

are the same. We also note that $P_{\chi}(\varphi)$ belongs to $\left(A\left(H_{U}\right) \otimes_{\mathbb{Z}} \mathbb{C}\right)^{\chi}$.

Let $\eta_{K}$ be the quadratic character of the extension $K / \mathbb{Q}$. Suppose that $\chi$ satisfies the self-duality condition $\omega_{A} \cdot \chi_{\mid \mathbb{A}_{\mathbb{Q}}^{\times}}=1$, where $(\cdot)_{\mid \mathbb{A}_{\mathbb{Q}}^{\times}}$ means restriction of the character $(\cdot)$ to the idele group $\mathbb{A}_{\mathbb{Q}}^{\times}$and $\omega_{A}$ is the central character of the automorphic representation $\pi_{A}$. We assume for simplicity that $\omega_{A}$ is trivial, and therefore $\chi_{\mid \mathbb{A}_{Q}^{\times}}=1$. For any place $v$ of $\mathbb{Q}$, let $\varepsilon\left(1 / 2, \pi_{A, v}, \chi_{v}\right) \in\{ \pm 1\}$ be the sign of the functional equation with respect to its center of symmetry $s=1 / 2$ of the local representation $\pi_{A, v} \otimes \chi_{v}$. Define the set

$$
\Sigma(A, \chi)=\left\{v \text { place of } \mathbb{Q}: \varepsilon\left(1 / 2, \pi_{A, v}, \chi_{v}\right) \neq \eta_{K, v}(-1)\right\} .
$$

Proposition 4.2. The real place $\infty$ belongs to the set $\Sigma(A, \chi)$, and every finite prime $p \in \Sigma(A, \chi)$ divides the conductor of $A$.

Proof: According to $[\mathbf{C V}$, Section 1], the real place $\infty$ belongs to the set $\Sigma(A, \chi)$ if $\chi_{\infty}=1$ and $\pi_{A, \infty}$ is the holomorphic discrete series (of weight at least 2). The first condition is true by our assumptions, while the second one holds because $\pi_{A}$ is the automorphic representation attached to an abelian variety. On the other hand, also from loc. cit. we know that if $p$ is a finite prime in the set $\Sigma(A, \chi)$, then $\pi_{A, p}$ is either special or supercuspidal, and therefore $p$ must divide the conductor of $A$.

Remark 4.3. If $p$ is a finite prime belonging to $\Sigma(A, \chi)$, one also knows that $K_{p}:=K \otimes_{\mathbb{Q}} \mathbb{Q}_{p}$ is a field. In particular, if $B$ is an indefinite quaternion algebra whose ramification set is supported in $\Sigma(A, \chi)$, then $K$ splits $B$ (i.e. $K$ embeds as a maximal subfield of $B$ ).

Let $\varepsilon\left(1 / 2, \pi_{A}, \chi\right)$ be the sign of the functional equation with respect to its center of symmetry $s=1 / 2$ of the global representation $\pi_{A} \otimes \chi$. Then

$$
\varepsilon\left(1 / 2, \pi_{A}, \chi\right)=(-1)^{|\Sigma(A, \chi)|} .
$$

Recall our assumption that the central character $\omega_{A}$ of $\pi_{A}$ is trivial, and let now $\chi$ be a character of $\operatorname{Gal}\left(K^{\mathrm{ab}} / K\right)$. Suppose that $\chi$ factors through $\operatorname{Gal}\left(H_{c} / K\right)$ where $H_{c}$ is the ring class field of conductor $c$; if there is no $c^{\prime} \mid c$ such that $\chi$ factors through $\operatorname{Gal}\left(H_{c^{\prime}} / K\right)$, we say that $\chi$ has conductor $c$; if $\chi$ factors through $\operatorname{Gal}\left(H_{c} / K\right)$, then the conductor of $\chi$ divides $c$. 
Suppose we have a character $\chi$ of conductor dividing the positive integer $c$ and a Heegner point $P_{c}$ of conductor $c$ in $X\left(H_{c}\right)$. For any $\varphi \in$ $\pi_{A}$ define

$$
P_{c, \varphi}^{\chi}:=\sum_{\sigma \in \operatorname{Gal}\left(H_{c} / K\right)} \varphi\left(P_{c}^{\sigma}\right) \otimes \chi(\sigma) .
$$

If $|\Sigma(A, \chi)|$ is odd, by [YZZZ, Theorem 1.3.1] one can choose $\varphi$ such that

$$
P_{c, \varphi}^{\chi} \neq 0 \text { in }\left(A\left(H_{c}\right) \otimes_{\mathbb{Z}} \mathbb{C}\right)^{\chi} \Longleftrightarrow L^{\prime}\left(\pi_{A}, \chi, 1 / 2\right) \neq 0 .
$$

From now on, we fix such a $\varphi$ and write simply $P_{c}^{\chi}$ for $P_{c, \varphi}^{\chi}$.

4.2. Euler systems and BSD conjecture. Before discussing our applications to the BSD conjecture, we recall the following result, which in this general form is due to Nekovář [Nek1].

Theorem 4.4 (Nekovář). Suppose that $A / \mathbb{Q}$ is a modular abelian variety of dimension d. Fix an imaginary quadratic field $K$ and an anticyclotomic character $\chi$ factoring through $H_{c}$ for some integer $c \geq 1$ such that the cardinality of $\Sigma(A, \chi)$ is odd. Let $B$ be the indefinite quaternion algebra of discriminant equal to the product of finite primes in $\Sigma(A, \chi)$. Assume that $A$ does not acquire $C M$ over any imaginary quadratic field contained in $H_{c}$, and that there exists

(1) an order $\mathcal{R}$ of $B$ with an uniformization $J_{\mathcal{R}}=\operatorname{Jac}\left(X_{\mathcal{R}}\right) \rightarrow A$ defined over $\mathbb{Q}$, and

(2) a Heegner point $P_{c}$ in $X_{\mathcal{R}}\left(H_{c}\right)$.

Then the following implication holds:

$$
\left.L^{\prime}\left(\pi_{A}, \chi, 1 / 2\right) \neq 0 \Longrightarrow \operatorname{dim}_{\mathbb{C}}\left(A\left(H_{c}\right) \otimes_{\mathbb{Z}} \mathbb{C}\right)^{\chi}\right)=d .
$$

We first observe that if the ramification set of the quaternion algebra $B$ coincides with $\Sigma(A, \chi)-\{\infty\}$, then there always exists a uniformization $J_{U}=\operatorname{Jac}\left(X_{U}\right) \rightarrow A$ for some open compact subgroup $U$ of $\hat{B}^{\times}$; so in (1) we are asking that this $U$ is associated with an order.

Theorem 4.5. Fix the following objects:

(1) a modular abelian variety $A / \mathbb{Q}$ of dimension $d$ and conductor $N^{d}$,

(2) an imaginary quadratic field $K$, and

(3) an anticyclotomic character $\chi$ factoring through the ring class field $H_{c}$ of $K$ of conductor $c \geq 1$ such that the cardinality of $\Sigma(A, \chi)$ is odd.

If $2 \in \Sigma(A, \chi)$ and $2^{3} \mid N$, assume that $\operatorname{val}_{2}(N)$ is odd. Let $B$ denote the indefinite quaternion algebra of discriminant $\Delta$ equal to the product of all the finite primes in $\Sigma(A, \chi)$. Then there exists an order $\mathcal{R}$ of type 
$T=\left(N_{\text {Eic }} ; N_{\text {Car }} ;\left\{\left(L_{p}, \nu_{p}\right)\right\}_{p \mid \Delta}\right)$ in $B$ and a Heegner point $P_{c^{\prime}} \in X_{\mathcal{R}}\left(H_{c^{\prime}}\right)$ with $c \mid c^{\prime}$ such that:

(1) $A$ is uniformized by $X_{\mathcal{R}}$, hence there is a surjective morphism $J_{\mathcal{R}} \rightarrow A$ defined over $\mathbb{Q}$;

(2) $N$ divides the level $N_{\text {Eic }} \cdot N_{\text {Car }}^{2} \cdot \prod_{p \mid \Delta} p^{\nu_{p}}$ of $\mathcal{R}$;

(3) $c$ divides $c^{\prime}$.

Proof: The problem is local, being equivalent to the existence of optimal local embeddings for all primes $\ell$. Fix the order $\mathcal{R}_{\min }$ of type $T_{\min }=$ $\left(N_{\text {Eic }} ; N_{\text {Car }} ;\left\{\left(L_{p}, \nu_{p}^{\prime}\right)\right\}_{p \mid \Delta}\right)$ and level $N_{\text {min }}=N_{\text {Eic }} N_{\text {Car }}^{2} N_{\Delta}$ as in the proof of Proposition 2.8, choosing the integers $N_{\text {Eic }}$ and $N_{\text {Car }}$ such that $N_{\text {Car }}$ is divisible only by primes $p$ which are inert in $K$ and do not divide $c$.

For primes $p \mid N_{\text {Eic }} N_{\text {Car }}$ which are split in $K$, one knows that the set of local optimal embeddings of the required form is non-empty (cf. Lemma 3.4).

Fix until the end of the proof a prime $p \mid N_{\min }$ which is inert or ramified in $K$. Let $m$ be the $p$-adic valuation of $c$ and set $n:=\operatorname{val}_{p}\left(N_{\min }\right)$. If $p$ divides $N_{\text {Car }}$, then we can apply Lemma 3.6 and show that the maximal order $R_{c} \otimes_{\mathbb{Z}} \mathbb{Z}_{p}$ embeds optimally into $\mathcal{R}_{\min } \otimes_{\mathbb{Z}} \mathbb{Z}_{p}$. So suppose from now on that $p$ does not divide $N_{\text {Car }}$.

Suppose first that $p \notin \Sigma(A, \chi)$. If $m \geq n / 2$ (unramified case) or $m \geq(n-1) / 2$ (ramified case) then Lemma 3.5 shows that the set of local optimal embeddings of $R_{c} \otimes_{\mathbb{Z}} \mathbb{Z}_{p}$ into $\mathcal{R}_{\text {min }} \otimes_{\mathbb{Z}} \mathbb{Z}_{p}$ is non-empty. If these conditions do not hold, replacing $m$ by $m^{\prime}$ such that $m^{\prime} \geq$ $n / 2$ (unramified case) or $m^{\prime} \geq(n-1) / 2$ (ramified case) then the local order $R_{c^{\prime}} \otimes_{\mathbb{Z}} \mathbb{Z}_{p}$ with $c^{\prime}=c \cdot p^{m^{\prime}-m}$ embeds optimally into $\mathcal{R}_{\min } \otimes_{\mathbb{Z}} \mathbb{Z}_{p}$.

Suppose now that $p \in \Sigma(A, \chi)$. Take any pair $\left(m^{\prime}, n^{\prime}\right)$ satisfying the following condition:

- if $n$ is odd, then $n^{\prime}=2 m^{\prime}+1$;

- if $n$ is even, then $n^{\prime}=2 m^{\prime}$ if $p$ is inert in $K$ whereas $n^{\prime}=2\left(m^{\prime}+1\right)$ if $p$ ramifies in $K$.

Choose also the pair $\left(m^{\prime}, n^{\prime}\right)$ so that $m^{\prime} \geq m$ and $n^{\prime} \geq n$. Comparing with the results recalled in $\S 3.2 .3$, we see that the set of optimal embeddings of the local quadratic order of conductor $p^{m^{\prime}}$ into the local quaternion order $R_{n^{\prime}}\left(L_{p}\right) \subseteq \mathcal{R}_{\min } \otimes_{\mathbb{Z}} \mathbb{Z}_{p}$ of type $\left(L_{p}, n^{\prime}\right)$ is non-zero.

Corollary 4.6. Let $A / \mathbb{Q}, K$, and $\chi$ be as in the previous theorem. If $A$ does not acquire $C M$ over any imaginary quadratic field contained in $H_{c}$ and $L^{\prime}\left(\pi_{A}, \chi, 1 / 2\right) \neq 0$, then $\left.\operatorname{dim}_{\mathbb{C}}\left(A\left(H_{c}\right) \otimes_{\mathbb{Z}} \mathbb{C}\right)^{\chi}\right)=d$. 
Proof: Let $\mathcal{R}$ and $c^{\prime}$ be as in the statement of Theorem 4.5 and apply Theorem 4.4, viewing $\chi$ as a character of $\operatorname{Gal}\left(H_{c^{\prime}} / K\right)$ via the canonical projection $\operatorname{Gal}\left(H_{c^{\prime}} / K\right) \rightarrow \operatorname{Gal}\left(H_{c} / K\right)$.

4.3. Proof of Theorem A. Theorems 4.4 and 4.5, although giving an Euler System which is sufficient for the proof of our main result in the introduction, are not completely satisfying in the sense that they are not effective in the computation of the order $\mathcal{R}$. Suppose we are in the situation of the theorem, so that we are given a modular abelian variety $A / \mathbb{Q}$, an imaginary quadratic field $K$, and an anticyclotomic character $\chi$ of conductor $c$ such that $\Sigma(A, \chi)$ has odd cardinality. Then we would like to have Heegner points in $\mathcal{R}_{\text {min }}$ for a choice of minimal parametrization $J_{\mathcal{R}_{\text {min }}} \rightarrow A$ described in Proposition 2.8, or at least of level $\mathcal{R}$ with $\mathcal{R} \subseteq \mathcal{R}_{\text {min }}$. We begin by discussing a couple of examples.

Example 4.7. Let $A=E$ be an elliptic curve of conductor $N=p^{2} q$, with $q$ and $p$ odd distinct primes both inert in $K$. Assume that the automorphic representation $\pi_{E}$ attached to $E$ is supercuspidal at $p$. Let $B$ be the quaternion algebra of discriminant $p q, \mathcal{R}=\mathcal{R}_{\text {min }}$ be the Hijikata-Pizer-Shemanske order $\mathcal{R}=\mathcal{R}_{\text {min }}$ of level $N=p^{2} q$ (and type $\left(1 ; 1 ;\left\{\left(L_{p}, 2\right),\left(L_{q}, 1\right)\right\}\right)$, for any choice of quadratic ramified extension $\left.L_{p} / \mathbb{Q}_{p}\right)$, and let $X_{\mathcal{R}}$ be its associated Shimura curve. Note that $K$ embeds into $B$ because both $p$ and $q$ are inert in $K$. Let $\chi$ be an anticyclotomic character of $p$-power conductor.

Consider first the case of the trivial character $\mathbf{1}$. Then $\varepsilon_{p}(E / K, \mathbf{1})=$ $+1[\mathbf{D e l 2},(5.5 .1)]$. Therefore $\Sigma(E, \mathbf{1})=\{q, \infty\}$ and in fact there are no Heegner points of conductor 1 in $X_{\mathcal{R}}$ (cf. $\S 3.2 .3$, case (1c)). The case of non-trivial conductor $p^{m}$ with $m \geq 2$ is similar: by [Tun, p. 1299] we know that $\varepsilon_{p}(E / K, \chi)=+1$, so $\Sigma(E, \mathbf{1})=\{q, \infty\}$ and in fact there are no Heegner points of conductor $p^{m}$ in $X_{\mathcal{R}}$. So the only case in which $\Sigma(E, \chi)=\{p, q, \infty\}$ may occur for a non-trivial character $\chi$ of conductor $p$, and by case (1c) in $\S 3.2 .3$, Heegner points of conductor $c=p$ do exist in $X_{\mathcal{R}}$. In this case, we realize a perfect matching between existence of Heegner points on Shimura curves, and local $\varepsilon$-signs of functional equations.

Example 4.8. As in the above example, let $A=E$ be an elliptic curve of conductor $N=p^{2} q$, with $q$ and $p$ odd distinct primes and suppose that $q$ is inert and $p$ is ramified in $K$. Identify the Weil-Deligne group $W_{\mathbb{Q}_{p}}$ of $\mathbb{Q}_{p}$ with $\mathbb{Q}_{p}^{\times}$via the reciprocity map $r_{\mathbb{Q}_{p}}$, normalized in such a way that $r_{\mathbb{Q}_{p}}(a)$ acts on $\overline{\mathbb{F}}_{p}$ by the character $x \mapsto x^{|a|}$, where $|\cdot|=|\cdot|_{p}$ is the $p$-adic absolute value satisfying $|p|=p^{-1}$. Assume that the automorphic 
representation $\pi_{E}$ attached to $E$ is supercuspidal at $p$, and write it as $\pi_{E, p}=\operatorname{Ind}_{W_{F}}^{W_{\mathbb{Q}_{p}}}(\psi)$ where $F / \mathbb{Q}_{p}$ is a quadratic extension with associated character $\eta$ and $\psi: W_{F}^{\mathrm{ab}} \rightarrow \mathbb{C}^{\times}$is a quasi-character not factoring through the norm map; then we have $\eta \psi=|\cdot|^{-1}$ as quasi-characters of $\mathbb{Q}_{p}^{\times}$. The above conditions force $\psi$ to have conductor equal to $1, p \equiv 3 \bmod 4$ and $\psi_{\mid \mathbb{Z}_{p}^{\times}}=\eta$ [Pac, Corollary 3.1]. Consider the quaternion algebra $B$ of discriminant $p q$, the Hijikata-Pizer-Shemanske order $\mathcal{R}$ of level $p^{2} q$ as in the previous example and its associated Shimura curve $X_{\mathcal{R}}$. Again $\pi_{E}$ admits a Jacquet-Langlands lift to $X_{\mathcal{R}}$. In this situation, there are no Heegner points of conductor $p^{m}$ with $m \geq 1$ in $X_{\mathcal{R}}$. So, if for a character $\chi$ of conductor $p^{m}$ we have $\Sigma(E, \chi)=\{p, q, \infty\}$, then we expect to find Heegner points in appropriate coverings of $X_{\mathcal{R}}$, but not in $X_{\mathcal{R}}$ itself.

The above examples motivate our discussion below, leading to the proof of (a slightly refined version of) Theorem A in the introduction.

Fix for the rest of the article an elliptic curve $E / \mathbb{Q}$ of conductor $N$, an imaginary quadratic field $K$ of discriminant $-D$, and a ring class character $\chi$ of conductor $c$ of $K$. Let $\Delta$ be the product of the finite primes in $\Sigma(E, \chi)$, which is assumed to have odd cardinality, and let $B$ be the quaternion algebra of discriminant $\Delta$. Fix also $\mathcal{R}:=\mathcal{R}_{\text {min }}$ to be the minimal order of type $T_{\min }=\left(N_{\text {Eic }} ; N_{\text {Car }} ;\left\{\left(L_{p}, \nu_{p}\right)\right\}_{p \mid \Delta}\right)$ as in Proposition 2.8, on which the Jacquet-Langlands lift to $B$ of the newform $f \in S_{2}\left(\Gamma_{0}(N)\right)$ associated with $E$ is realized, and let $N_{\mathcal{R}}=N_{\text {Eic }} N_{\text {Car }}^{2} N_{\Delta}$ be its level. We can further assume the (coprime) integers $N_{\text {Eic }}$ and $N_{\text {Car }}$ satisfy that, for every prime $p$ dividing $N_{\text {Eic }} N_{\text {Car }}$,

$p \mid N_{\text {Car }}$ if and only if $p$ is inert in $K, \operatorname{val}_{p}(N)$ is even and $p \nmid c$.

From now on, we shall make the following assumption. Observe that under the hypotheses in Theorem A the assumption below is obviously satisfied (cf. Definitions 4.17, 4.18).

Assumption 4.9. Let $f \in S_{2}\left(\Gamma_{0}(N)\right)$ be the newform attached to the elliptic curve $E$ by modularity. With the above notations, the following conditions are assumed.

(i) Let $\pi_{f, p}$ be the $p$-component of the automorphic representation attached to $f$. Then $\pi_{f, p}$ has minimal Artin conductor among its twists by quasi-characters of $\mathbb{Q}_{p}^{\times}$in the following cases: (1) $p=2$; (2) $p \mid N_{\text {Eic }}$, with $\operatorname{val}_{p}(N)$ even and $p$ ramified in $K$; (3) $p=3$, with $3 \mid \Delta$ and $\operatorname{val}_{3}(N)=4$.

(ii) If $2^{3} \mid N_{\text {Eic }}$, then either 2 splits in $K$ or $\operatorname{val}_{2}\left(N_{\text {Eic }}\right)$ is odd and 2 is inert in $K$. 
(iii) If $2 \mid \Delta$ and $2^{3} \mid N$, then $\operatorname{val}_{2}(N)$ is odd; if in addition $\pi_{f, 2}$ is supercuspidal, then 2 is inert in $K$.

(iv) If $\operatorname{val}_{3}\left(N_{\text {Eic }}\right)=4$ and 3 is inert in $K$, then $\operatorname{val}_{3}(c) \neq 1$.

(v) If $3 \mid \Delta, \operatorname{val}_{3}(N)=3$ or 5 and 3 is ramified in $K$, then $\operatorname{val}_{3}(c) \geq$ $\left(\operatorname{val}_{3}(N)-1\right) / 2$.

Remark 4.10. As commented in Remark 2.7, if $2 \mid \Delta$ and $\operatorname{val}_{2}(N)$ is even and greater than or equal to 4, then [HPS2, Theorem 3.9] claims that $f$ is a twist of a modular form of lower level, but such statement is not true in general. One expects that those cases for which that assertion holds can be dealt with via different methods. If $f=g \otimes \xi$, then one expects to construct points on the modular abelian varieties attached to $g$, and then, using twisting techniques, to construct points on the elliptic curve. It seems possible that condition (i) can be treated by similar considerations.

Write also $R_{c}$ for the order of conductor $c$ in $K$ of conductor $c$ as usual. Our goal now is to investigate under which conditions $R_{c}$ embeds optimally into $\mathcal{R}$. And in those cases where this does not happen, we must find a suitable suborder $\mathcal{R}^{\prime}$ of $\mathcal{R}$ such that $R_{c}$ does optimally embed into it. This task is carried out in a series of lemmas below.

The problem is clearly local, and it suffices to study it at those primes dividing $N$. So fix from now on a prime $p \mid N$, and set the following notations. We write $m:=\operatorname{val}_{p}(c)$ for the $p$-adic valuation of $c$, and $n:=\operatorname{val}_{p}\left(N_{\mathcal{R}}\right)$ for that of $N_{\mathcal{R}}$. By the discussion prior to Proposition 2.8, observe that $n$ coincides with $\operatorname{val}_{p}(N)$. Then we denote by $\mathcal{E}_{p}(m, n)$ the set of (local) optimal embeddings of $R_{c} \otimes_{\mathbb{Z}} \mathbb{Z}_{p}$ into $\mathcal{R} \otimes_{\mathbb{Z}} \mathbb{Z}_{p}$. Recall that the conditions that characterize the non-emptiness of $\mathcal{E}_{p}(m, n)$, in each of the possible cases, have been collected in $\S 3.2$. If $p$ is not split in $K$, then we write $\chi_{p}$ for the component of $\chi$ at the unique prime of $K$ above $p$. In that case, notice that $m=c\left(\chi_{p}\right)$, the (exponent of the) conductor of $\chi_{p}$.

If the prime $p$ does not belong to $\Sigma(E, \chi)$ (i.e., if $p \nmid \Delta)$, then we will prove in Lemma 4.11 that $\mathcal{E}_{p}(m, n) \neq \emptyset$, hence we do not need to increase the level of $\mathcal{R}$ at $p$. If in contrast $p \mid \Delta$, and $n^{\prime} \geq n$ is an integer (with the same parity as $n$ ), then we define $\mathcal{R}^{\prime}$ to be the suborder of $\mathcal{R}$ obtained by replacing the local data $\left(L_{p}, n\right)$ at $p$ in the type of $\mathcal{R}$ by the data $\left(L_{p}, n^{\prime}\right)$. In the series of Lemmas 4.12 to 4.15 we prove that one can always choose such a suborder $\mathcal{R}^{\prime}$ of $\mathcal{R}$ for which $\mathcal{E}_{p}\left(m, n^{\prime}\right) \neq \emptyset$. For convenience of the reader, we summarize in Table 1 the minimal choice for $n^{\prime}$ in each of the possible cases, depending on the local data at $p$, 
when $p \neq 2,3$. The third column indicates the condition on $m$ (if any) imposed by the fact that $p \in \Sigma(E, \chi)$.

\begin{tabular}{|c|c|c|c|}
\hline$\pi_{E, p}$ & $p, n$ & restrictions on $m$ & $n^{\prime}$ \\
\hline \multirow{2}{*}{ supercuspidal } & $p$ inert & $m \geq 1$ & $2 m$ \\
& $p$ ramified & & $2(m+1)$ \\
\hline \multirow{3}{*}{ Steinberg } & $p$ inert, $n=1$ & $m=0$ & $n$ \\
& $p$ inert, $n=2$ & $m=1$ & $n$ \\
& $p$ ramified, $n=1$ & & $2 m+1$ \\
& $p$ ramified, $n=2$ & & $2(m+1)$ \\
\hline
\end{tabular}

TABLE 1. Choice of $n^{\prime}$ for primes $p \in \Sigma(E, \chi), p \neq 2,3$.

As announced above, first we consider the case where $p$ does not belong to the set $\Sigma(E, \chi)$, so that $B$ is split at $p$.

Lemma 4.11. If $p \notin \Sigma(E, \chi)$, then $\mathcal{E}_{p}(m, n) \neq \emptyset$.

Proof: First observe that if $p \notin \Sigma(E, \chi)$ then $p$ divides $N_{\text {Eic }} N_{\text {Car }}$. Having said this, notice that if $p \mid N_{\text {Car }}$ then $\mathcal{E}_{p}(m, n)$ is non-empty by Lemma 3.6 (because if $p \mid N_{\text {Car }}$ then $m=0$ ). So we assume for the rest of the proof that $p$ divides $N_{\text {Eic }}$. By our choice of $N_{\text {Eic }}$ and $N_{\text {Car }}^{2}$, we shall distinguish three cases:

(1) $p$ is split in $K$;

(2) $p$ is inert or ramified in $K$ and $n$ is odd;

(3) $p$ is inert or ramified in $K$ and $n$ is even.

If $p$ is in case (1), then Lemma 3.4 implies that $\mathcal{E}_{p}(m, n)$ is nonempty. Suppose that $p$ is in case (2), and assume first that $n=1$, which is the only possible value if $p \geq 5$. If $p$ ramifies in $K$, then $\mathcal{E}_{p}(m, n) \neq \emptyset$ by part (2) in Lemma 3.5. If $p$ is inert in $K$, then we split the discussion according to whether $p \nmid c$ or $p \mid c$. In the former case, $\varepsilon_{p}(E / K, \chi)=\varepsilon_{p}(E / K, 1)=-1$ but $\eta_{K, p}(-1)=1$, thus $p$ should be in $\Sigma(E, \chi)$, contradicting our hypotheses. And in the latter case, we have $m \geq 1$ and therefore $2 m \geq n=1$, hence Lemma 3.5 shows that $\mathcal{E}_{p}(m, n) \neq \emptyset$. Thus we are left with the cases where $p=2$ or 3 and $n=\operatorname{val}_{p}(N)>1$ is odd.

- If $p=3$, then $n$ can be either 3 or 5 . Then $\pi_{E, 3}$ is supercuspidal induced from a quasicharacter $\psi$ of conductor $n-1$ of a ramified quadratic extension $F_{3}$ of $\mathbb{Q}_{3}$. If 3 is inert in $K$, we know on the one hand by Lemma 3.5 that $\mathcal{E}_{3}(m, n) \neq \emptyset$ if and only if $m \geq n / 2$, hence if and only if $m>(n-1) / 2$. On the other hand, being 3 inert in $K$ the assumption that $3 \notin \Sigma(E, \chi)$ tells us that $\varepsilon_{3}(E / K, \chi)=1$ and by [Tun, Proposition 2.8] this holds if and only if $m>(n-1) / 2=$ 
1. Thus it follows that $\mathcal{E}_{3}(m, n) \neq \emptyset$. Now suppose that 3 ramifies in $K$. Then $\eta_{K, 3}(-1)=-1$, hence $\varepsilon_{3}(E / K, \chi)=-1$ because $3 \notin \Sigma(E, \chi)$. By Lemma 3.5 we have that $\mathcal{E}_{3}(m, n) \neq \emptyset$ if and only if $m \geq(n-1) / 2$. By [Tun, Proposition 2.8], if $m<(n-1) / 2$ then $\varepsilon_{3}(E / K, \chi)=+1$, therefore we must have $m \geq(n-1) / 2$, and we conclude that $\mathcal{E}_{3}(m, n) \neq \emptyset$.

- If $p=2$, then $n$ can be either 3,5 , or 7. By Assumption 4.9 (ii), we may suppose that 2 is inert in $K$, so that $2 \notin \Sigma(E, \chi)$ implies that $\varepsilon_{2}(E / K, \chi)=1$. Further, by Assumption 4.9 (i), we may assume that $\pi_{E, 2}$ has minimal conductor among its twists as well.

Suppose first that $n=3$. Then [Tun, Proposition 3.7] implies that $m \geq 2$, and then by part (1) of Lemma 3.5 we deduce that $\mathcal{E}_{2}(m, n) \neq \emptyset$. If $n=5$, then $\pi_{E, 2}$ is supercuspidal induced from a quasicharacter of conductor 3 on a ramified extension of $\mathbb{Q}_{2}$ with discriminant valuation 2. If the conductor of $\chi$ were $m<3$, then [Tun, Lemma 3.2] would imply that $\varepsilon_{2}(E / K, \chi)=-1$, thus we deduce that $m \geq 3$. And then by part (1) of Lemma 3.5 we conclude that $\mathcal{E}_{2}(m, n) \neq \emptyset$.

Lastly, if $n=7$ then $\pi_{E, 2}$ is supercuspidal of exceptional type, and its conductor is minimal with respect to twist. Then $\varepsilon_{2}(E / K, \chi)=$ 1 implies, by [Tun, Lemma 3.2], that $m \geq 4$. But then we deduce that $\mathcal{E}_{2}(m, n) \neq \emptyset$ thanks to Lemma 3.5, part (1).

Finally, suppose that $p$ is in case (3). Again let us start with the case $n=2$, which is the only possible case if $p \geq 5$. If $p$ is inert in $K$, then our choice of $N_{\text {Eic }}$ and $N_{\text {Car }}$ implies that $m=\operatorname{val}_{p}(c) \geq 1$, hence we see that $2 m \geq n$ and Lemma 3.5 implies that $\mathcal{E}_{p}(m, n) \neq \emptyset$. If $p$ is ramified, Assumption 4.9 (i) implies that $\pi_{E}$ is supercuspidal at $p$. If it were $m=0$, then [Tun, Lemma 3.2] would imply that $p \in \Sigma(E, \chi)$, hence we have $m \geq 1$ and Lemma 3.5 implies again that $\mathcal{E}_{p}(m, n) \neq \emptyset$. We are then left with the cases where $p=2$ or 3 , and $n=\operatorname{val}_{p}(N)>2$ is even.

By Assumption 4.9 (ii), the case $p=2$ does not arise, so we assume that $p=3$. Then the only possible value for $n$ is 4 . If 3 is inert in $K$, then Assumption 4.9 (iv) implies that $m \geq 2$, and by part (1) of Lemma 3.5 we conclude that $\mathcal{E}_{3}(m, n) \neq \emptyset$. If 3 ramifies in $K$, then it follows from condition (i) in Assumption 4.9 that $\pi_{E, 3}$ is supercuspidal induced from a quasicharacter of conductor 2 of the unramified quadratic extension of $\mathbb{Q}_{3}$. Then [Tun, Lemma 3.2] implies that if $m \leq 3$ then 3 would belong to $\Sigma(E, \chi)$. Thus we have $m \geq 4$ and by Lemma 3.5 (2) it follows that $\mathcal{E}_{3}(m, n) \neq \emptyset$. 
Next we will deal with the case that $p \in \Sigma(E, \chi)$, or equivalently $p \mid \Delta$. This means that $\varepsilon_{p}(E / K, \chi)=-\eta_{K, p}(-1)$. So if $p$ is odd, then

$$
\varepsilon_{p}(E / K, \chi)=\left\{\begin{array}{lll}
-1 & \text { if } p \text { is inert in } K \\
-1 & \text { if } p \text { is ramified in } K \text { and } p \equiv 1 \quad \bmod 4 \\
1 & \text { if } p \text { is ramified in } K \text { and } p \equiv 3 \quad \bmod 4
\end{array}\right.
$$

Let $\pi_{E}$ be the automorphic representation attached to $E$, and $\pi_{E, p}$ be its $p$-th component. The (exponent of the) conductor of $\pi_{E, p}$ is $\operatorname{val}_{p}(N)$.

We will split our discussion into distinct lemmas, to distinguish between the cases where $\pi_{E, p}$ is supercuspidal or Steinberg. If $\pi_{E, p}$ is supercuspidal, then it is well-known that $\operatorname{val}_{p}(N) \geq 2$. For $p \geq 5$ this means that $\operatorname{val}_{p}(N)=2$, whereas for $p=3$ (resp. $p=2$ ) we have $2 \leq \operatorname{val}_{3}(N) \leq 5$ (resp. $2 \leq \operatorname{val}_{2}(N) \leq 8$ ). Besides, if $\pi_{E, p}$ is Steinberg, then $\operatorname{val}_{p}(N)$ can only be 1 or 2 if $p$ is odd, whereas if $p=2$ then $\operatorname{val}_{2}(N) \in\{1,4,6\}$. However, the reader should keep in mind that under Assumption 4.9, some of the previous cases with $p=2$ do not appear in our discussion.

Lemma 4.12. If $p \in \Sigma(E, \chi), \pi_{E}$ is supercuspidal at $p$ and $p$ is inert in $K$, then there exists $n^{\prime} \geq n$ such that $\mathcal{E}_{p}\left(m, n^{\prime}\right) \neq \emptyset$.

Proof: The assumptions $p \in \Sigma(E, \chi)$ and $p$ inert in $K$ imply that $\varepsilon_{p}(E / K, \chi)=-1$. Suppose first that $p$ is odd. We have the following cases:

(1) $n=2$. If $m=0$, then $\varepsilon_{p}(E / K, \chi)=1$ by [Del2, (5.5.1)], so we may assume that $m \geq 1$. But then defining $n^{\prime}:=2 m \geq n$ we conclude by case $(1 \mathrm{c})$ in $\S 3.2 .3$ that $\mathcal{E}_{p}\left(m, n^{\prime}\right) \neq \emptyset$.

(2) $p=3$ and $n>2$. In this case $n=\operatorname{val}_{3}(N)$ can be either 3,4 , or 5 . If $n \neq 4$, then $\pi_{E, p}$ is induced from a quasicharacter $\psi$ of conductor $n-1$ of a ramified quadratic extension of $\mathbb{Q}_{3}$. On the one hand, from $\S 3.2 .3$, case (1a) we see that $\mathcal{E}_{3}(m, n) \neq \emptyset$ if and only if $m \leq(n-1) / 2$. And on the other hand, by [Tun, Proposition 2.8] one has that $\varepsilon_{3}(E / K, \chi)=-1$ if and only if $m \leq(n-1) / 2$. Thus we conclude that $\mathcal{E}_{3}(m, n) \neq \emptyset$.

Suppose now that $n=4$. In this case, $\pi_{E, 3}$ is induced from a quasicharacter $\psi$ of conductor 2 of the unramified quadratic extension of $\mathbb{Q}_{3}$. If $\chi_{p}$ is unramified, that is $m=0$, then by [Del2, (5.5.1)] we would have $\varepsilon_{p}(E / K, \chi)=1$, hence it must be $m \geq 1$. However, if $m=1$ then by Assumption 4.9 (i) we can use [Tun, Proposition 3.5] to show that $\varepsilon_{3}(E / K, \chi)=1$, thus it follows that $m \geq 2$. But then for $n^{\prime}:=2 m \geq n$ we have that $\mathcal{E}_{3}\left(m, n^{\prime}\right) \neq \emptyset$ by case $(1 \mathrm{c})$ in $\S 3.2 .3$. 
Now we assume that $p=2$. Again we can split the discussion into cases.

(i) First suppose $n=\operatorname{val}_{p}(N)=2$. As above, if $m=0$ then $\varepsilon_{p}(E / K, \chi)=$ 1 , hence it must be $m \geq 1$. Letting $n^{\prime}:=2 m \geq n$, case (2b) now ensures that $\mathcal{E}_{p}\left(m, n^{\prime}\right) \neq \emptyset$.

(ii) If $\operatorname{val}_{p}(N)>2$, then Assumption 4.9 (iii) implies that $n$ is odd. If $n=3$, on the one hand by [Tun, Proposition 3.7] we have that $\varepsilon_{2}(E / K, \chi)=-1$ if and only if $m \leq 1$. And on the other hand, case (2f) in $\S 3.2 .3$ tells us that $\mathcal{E}_{2}(m, n) \neq \emptyset$ if and only if $m \leq 1$, thus we conclude that $\mathcal{E}_{2}(m, n) \neq \emptyset$. If $n$ is either 5 or 7 , again according to $\S 3.2 .3$ case $(2 \mathrm{f})$ we see that if $m \leq(n-1) / 2$ then $\mathcal{E}_{2}(m, n) \neq \emptyset$. If not, defining $n^{\prime}:=2 m+1$ we will have $\mathcal{E}_{2}\left(m, n^{\prime}\right) \neq \emptyset$.

This concludes the proof.

Lemma 4.13. If $p \in \Sigma(E, \chi), \pi_{E}$ is supercuspidal at $p$ and $p$ is ramified in $K$, then there exists $n^{\prime} \geq n$ such that $\mathcal{E}_{p}\left(m, n^{\prime}\right) \neq \emptyset$.

Proof: As in the previous lemma, we assume first that $p$ is odd. We have the following cases:

(1) Suppose $n=\operatorname{val}_{p}(N)=2$. If $m=0$, we deduce from $\S 3.2 .3$ (cases $(1 \mathrm{~d})$ and $(1 \mathrm{e}))$ that $\mathcal{E}_{p}(m, n) \neq \emptyset$. In contrast, if $m>0$ the set $\mathcal{E}_{p}(m, n)$ is empty. But by virtue of $\S 3.2 .3$, cases $(1 \mathrm{~d})$ and $(1 \mathrm{e})$, for $n^{\prime}:=2(m+1)>n$ we have $\mathcal{E}_{p}\left(m, n^{\prime}\right) \neq \emptyset$.

(2) Suppose that $p=3$ and $n=\operatorname{val}_{3}(N) \geq 3$. In this case, $3 \leq n \leq 5$. Assume first that $n=4$. In this case, the quadratic extension $L_{3} / \mathbb{Q}_{3}$ is ramified. Up to replacing $L_{3}$ by the other quadratic ramified extension, we might assume that $K_{3} \nsucceq L_{3}$. Then from case (1e) in $\S 3.2 .3$ we see that $\mathcal{E}_{p}(m, n) \neq \emptyset$ if and only if $m \leq 1$. If $m>1$, then we take $n^{\prime}:=2(m+1)$, and again case (1e) in $\S 3.2 .3$ tells us that $\mathcal{E}_{p}\left(m, n^{\prime}\right) \neq \emptyset$. If $n=3$ or 5 instead, then Assumption $4.9(\mathrm{v})$ implies that $m \geq(n-1) / 2$. By setting $n^{\prime}:=2 m+1 \geq n$, we obtain $\mathcal{E}_{p}\left(m, n^{\prime}\right) \neq \emptyset$ by case $(1 \mathrm{~b})$ in $\S 3.2 .3$.

Now we deal with the case $p=2$. By Assumption 4.9 (iii), if it were $\operatorname{val}_{2}(N)>2$ then 2 should be inert in $K$, thus we only need to consider the case $n=\operatorname{val}_{2}(N)=2$. By cases (2c), (2d), and (2e) in $\S 3.2 .3$ we have that $\mathcal{E}_{p}(m, n) \neq \emptyset$ if and only if $m=0$. Notice first that $\pi_{E, p}$ is of minimal conductor among its twists, since supercuspidal representations have conductor $\geq 2$. Then, by virtue of [Tun, Proposition 3.5], we see that $\varepsilon_{p}(E / K, \chi) \eta_{K, 2}(-1)$ is +1 (resp. -1 ) if and only if $m \geq 2$ (resp. $m<2$ ). But the hypothesis that $2 \in \Sigma(E, \chi)$ tells us that $\varepsilon_{p}(E / K, \chi) \eta_{K, 2}(-1)=-1$, hence $m<2$. But notice that $m$ cannot be 1 
in the case at hand, thus we deduce that $m=0$, and hence $\mathcal{E}_{p}(m, n) \neq \emptyset$ as desired.

Next we consider the Steinberg case. Write $\pi_{E, p}=\mathrm{Sp}_{2} \otimes \psi$ where $\psi: W_{\mathbb{Q}_{p}}^{\text {ab }} \rightarrow \mathbb{C}^{\times}$is a quadratic character. By [Tun, Proposition 1.7], we know $p \in \Sigma(E, \chi)$ if and only if $\chi_{p}^{-1}=\psi \circ \mathrm{Nr}$, where $x \mapsto \operatorname{Nr}(x)$ is the norm map from $K_{p}=K \otimes_{\mathbb{Q}} \mathbb{Q}_{p}$ to $\mathbb{Q}_{p}$.

Lemma 4.14. If $p \in \Sigma(E, \chi), \pi_{E}$ is Steinberg at $p$ and $p$ is inert in $K$, then there exists $n^{\prime} \geq n$ such that $\mathcal{E}_{p}\left(m, n^{\prime}\right) \neq \emptyset$.

Proof: By the above discussion, $p \in \Sigma(E, \chi)$ if and only if $\chi_{p}^{-1}=\psi \circ \mathrm{Nr}$. We assume first that $p$ is odd, so that $n=\operatorname{val}_{p}(N)$ can be either 1 or 2 . We split the discussion into subcases:

(1) $n=1$. Comparing with $\S 3.2 .3$ (1a), we see that $\mathcal{E}_{p}(m, n) \neq \emptyset$ if and only if $m=0$. On the other hand, $\psi$ is unramified and therefore if $p \in \Sigma(E, \chi)$ then $m=0$.

(2) $n=2$. Looking now at $\S 3.2 .3(1 \mathrm{c}), \mathcal{E}_{p}(m, n) \neq \emptyset$ if and only if $m=1$. On the other hand, $\psi$ is ramified with conductor equal to 1 , and therefore if $p \in \Sigma(E, \chi)$ then $m=1$.

Assume now that $p=2$. A priori one could have $\operatorname{val}_{2}(N) \in\{1,4,6\}$, but by condition (iii) in Assumption 4.9 we only need to deal with the case $\operatorname{val}_{2}(N)=1$. In this case, the character $\psi$ is unramified, and then since $2 \in \Sigma(E, \chi)$ we deduce that $m=0$. On the other hand, by case $(2 \mathrm{a})$ in $\S 3.2 .3$ we also have $\mathcal{E}_{p}(m, n) \neq \emptyset$ if and only if $m=0$. Thus $\mathcal{E}_{p}(m, n) \neq \emptyset$ as we want.

Lemma 4.15. If $p \in \Sigma(E, \chi), \pi_{E}$ is Steinberg at $p$ and $p$ is ramified in $K$, then there exists $n^{\prime} \geq n$ such that $\mathcal{E}_{p}\left(m, n^{\prime}\right) \neq \emptyset$.

Proof: Suppose first that $p$ is odd, so that $n=\operatorname{val}_{p}(N)$ is either 1 or 2 . Again by the above discussion, $p \in \Sigma(E, \chi)$ if and only if $\chi_{p}^{-1}=\psi \circ \mathrm{Nr}$. We have the following cases:

(1) $n=1$. If $m=0$, by case (1b) in $\S 3.2 .3$ we see that $\mathcal{E}_{p}(m, n) \neq \emptyset$. Otherwise, we can take $n^{\prime}:=2 m+1$, and again case (1b) in $\S 3.2 .3$ implies $\mathcal{E}_{p}\left(m, n^{\prime}\right) \neq \emptyset$.

(2) $n=2$. Again, for $m=0$ we have $\mathcal{E}_{p}(m, n) \neq \emptyset$ by cases (1d) or (1e) in $\S 3.2 .3$. If instead $m>0$, then we consider $n^{\prime}:=2(m+1)$ and by applying $\S 3.2 .3$, cases $(1 \mathrm{~d})$ and $(1 \mathrm{e})$, we see that $\mathcal{E}_{p}\left(m, n^{\prime}\right) \neq \emptyset$.

Now assume that $p=2$. As in the previous lemma, by Assumption 4.9 we only need to deal with the case $n=\operatorname{val}_{2}(N)=1$. Then notice that $\psi$ is unramified. On the other hand, now the hypothesis that 2 
belongs to $\Sigma(E, \chi)$ implies that either $\eta_{K, 2}(-1)=1$ and $\chi_{p}^{-1}=\psi \circ \mathrm{Nr}$ or $\eta_{K, 2}(-1)=-1$ and $\chi_{p}^{-1} \neq \psi \circ \mathrm{Nr}$. Having this into account, if $\eta_{K, 2}(-1)=$ 1 then the equality $\chi_{p}^{-1}=\psi \circ \mathrm{Nr}$ implies that $m=0$. By case $(2 \mathrm{a})$ in $\S 3.2 .3$ it thus follows that $\mathcal{E}_{p}(m, n) \neq \emptyset$. And if $\eta_{K, 2}(-1)=-1$, it could be the case that $m>0$. But in any case, defining $n^{\prime}:=2 m+1 \geq n=1$ case $(2 \mathrm{~g})$ in $\S 3.2 .3$ implies that $\mathcal{E}_{p}\left(m, n^{\prime}\right) \neq \emptyset$.

Combining the above lemmas, we obtain the following:

Theorem 4.16. Let $E / \mathbb{Q}$ be an elliptic curve of conductor $N, K$ be an imaginary quadratic field, and $\chi$ be an anticyclotomic character of conductor c. Suppose that the set $\Sigma(E, \chi)$ has odd cardinality, so that $\varepsilon(E / K, \chi)=-1$ and hence $L(E / K, \chi, 1)=0$. If Assumption 4.9 holds, then the set of Heegner points in $E\left(H_{c}\right)$ is non-empty. And if further $E$ does not acquire $C M$ over any imaginary quadratic field contained in $H_{c}$ and $L^{\prime}(E / K, \chi, 1) \neq 0$, then $\operatorname{dim}_{\mathbb{C}}\left(E\left(H_{c}\right) \otimes \mathbb{C}_{\mathbb{Z}}\right)^{\chi}=1$.

Proof: Let $B$ be the indefinite quaternion algebra ramified exactly at the finite primes in $\Sigma(E, \chi)$, and let $\mathcal{R}_{\text {min }}$ be the order in $B$ from Proposition 2.8. The above lemmas together imply that there is a suborder $\mathcal{R}$ of $\mathcal{R}_{\text {min }}$ such that the set of Heegner points of conductor $c$ in $X_{\mathcal{R}}\left(H_{c}\right)$ is non-empty. The Jacobian of $X_{\mathcal{R}}$ uniformizes $E$ as well, hence the set of Heegner points of conductor $c$ in $E\left(H_{c}\right)$ is non-empty. By Theorem 4.4, if $E$ does not acquire $\mathrm{CM}$ over any imaginary quadratic field contained in $H_{c}$, then $\operatorname{dim}_{\mathbb{C}}\left(E\left(H_{c}\right) \otimes \mathbb{C}_{\mathbb{Z}}\right)^{\chi}=1$.

We state now the above result in a more restrictive but maybe more attractive form, which already generalizes Theorem $\mathrm{A}$ in the introduction, by introducing a couple of definitions.

Definition 4.17. Let $p$ be a prime. We say that $f \in S_{2}\left(\Gamma_{0}(N)\right)$ has $p$-minimal Artin conductor if the $p$-component $\pi_{f, p}$ of the automorphic representation $\pi_{f}$ attached to $f$ has minimal conductor among its twists by quasi-characters of $\mathbb{Q}_{p}^{\times}$; in other words, if we write $a\left(\pi_{f}\right)$ for the Artin conductor of the automorphic representation $\pi_{f}$, we require that $a\left(\pi_{f}\right) \leq a\left(\pi_{f} \otimes \eta\right)$ for all quasi-characters $\eta$ of $\mathbb{Q}_{p}^{\times}$.

Requiring that $f \in S_{2}\left(\Gamma_{0}(N)\right)$ has $p$-minimal Artin conductor is actually equivalent to asking that the modular form $f$ is $p$-primitive, in the following sense (cf. [AL, p. 236] and [Piz2, Definition 8.6]): 
Definition 4.18. We say that a form $f \in S_{2}\left(\Gamma_{0}(N)\right)$ is p-primitive if $\operatorname{val}_{p}(N)$ is minimal among all the twists of $f$ by Dirichlet characters of $p$-power conductor. More generally, $f$ is said to be primitive if $f \neq g \otimes \xi$ for any Dirichlet character $\xi$ and any $g \in S_{2}\left(\Gamma_{0}(M)\right)$ with $M \neq N$.

Corollary 4.19. Let $E / \mathbb{Q}$ be an elliptic curve of conductor $N$, and $f \in S_{2}\left(\Gamma_{0}(N)\right)$ be the newform attached to $E$ by modularity. Let $K$ be an imaginary quadratic field, $\chi$ be an anticyclotomic character of conductor $c$, and suppose that the set $\Sigma(E, \chi)$ has odd cardinality. Assume the following conditions hold:

(1) $f$ is $p$-primitive at $p=2$ and at every prime $p \mid N$ with $\operatorname{val}_{p}(N)$ even;

(2) if $2^{3} \mid N$, then either 2 splits in $K$ or $\operatorname{val}_{2}(N)$ is odd and 2 is inert in $K$;

(3) if $\operatorname{val}_{3}(N) \geq 3$, then $\operatorname{val}_{3}(c) \geq\left(\operatorname{val}_{3}(N)-1\right) / 2$.

Then the set of Heegner points in $E\left(H_{c}\right)$ is non-empty. If further $E$ does not acquire $C M$ over any imaginary quadratic field contained in $H_{c}$ and $L^{\prime}(E / K, \chi, 1) \neq 0$, then $\operatorname{dim}_{\mathbb{C}}\left(E\left(H_{c}\right) \otimes \mathbb{C}_{\mathbb{Z}}\right)^{\chi}=1$.

Proof: We only need to remark that condition (1) implies condition (i) in Assumption 4.9, whereas condition (2) (resp. (3)) implies that both conditions (ii), (iii) (resp. (iv), (v)) in Assumption 4.9 hold.

4.4. Final remarks. It might be interesting to discuss how to extend the above theorem to the general case of abelian varieties. One can easily show that if $A / \mathbb{Q}$ is a modular variety of dimension $d$ and conductor $N^{d}$, and no prime divides $N$ to a power greater than 3 , then the argument for elliptic curves developed in the previous section also works for abelian varieties. However, it is easy to construct examples in which we do not have Heegner points in any cover of $X_{\mathcal{R}_{\text {min }}}$ if we allow the conductor of $A$ to be divisible by arbitrary powers of $p$ if we just consider orders of type $\left(N_{\text {Eic }} ; N_{\text {Car }} ;\left\{\left(L_{p}, \nu_{p}\right)\right\}\right)$, as the following examples show:

Example 4.20. Let $A / \mathbb{Q}$ be a modular abelian variety of conductor $N^{d}$, and suppose $N=p^{n} q$ with $p$ and $q$ distinct odd primes and $n=2 \varrho+1$ an odd integer. Let $\chi$ be a character of conductor $p^{m}$ with $m \geq 1$. Suppose that $p$ is ramified in $K$ and $q$ is inert in $K$. Then $q \in \Sigma(A, \chi)$. Now assume that $n$ is minimal among the conductor of all twists of $\pi_{A, p}$. In this case [Tun, Proposition 3.5] shows that if $m \leq n-1$ then $p \in \Sigma(A, \chi)$. If now $m<(n-1) / 2$, then comparing with $\S 3.2 .3$ we see that there are no Heegner points of conductor $p^{m}$ in any cover of $X_{\mathcal{R}_{\text {min }}}$ associated with an order as in Definition 1.3. 
Example 4.21. As in the above example, let $A / \mathbb{Q}$ be a modular abelian variety of conductor $N^{d}$, and suppose now that $N=p^{n} q$ with $p$ and $q$ two odd primes and $n=2 \varrho \geq 4$ an even integer. Let $\chi$ be a character of conductor $p^{m}$ with $m \geq 1$. Suppose first that $p \in \Sigma(A, \chi)$, so $\varepsilon_{p}(A, \chi)=-1$, and $q$ is inert in $K$, so $q \in \Sigma(A, \chi)$. Consider the quaternion algebra $B$ of discriminant $p q$ and the order $\mathcal{R}_{\text {min }}$ of $B$ and form the corresponding Shimura curve $X_{\mathcal{R}_{\text {min }}}$. From $\S 3.2 .3$, we see that if $m<n / 2$, then there are no Heegner points of conductor $p^{m}$ in any covering of $X_{\mathcal{R}_{\text {min }}}$ associated with special orders as in Definition 1.3. Secondly, suppose $p \notin \Sigma(A, \chi)$, so $\varepsilon_{p}(A, \chi)=+1$ and $q$ is split in $K$, so $q \notin \Sigma(A, \chi)$. In this case, if $m<n / 2$ then again there are no Heegner points of conductor $p^{m}$ in any cover of the Shimura curve $X_{\mathcal{R}_{\min }}$ associated with Eichler orders (which, in this case, correspond to modular curves and usual congruence subgroups).

As we may see from the above examples, it seems to us that that one should introduce more general type of orders to find other sources of Heegner points defined over the predicted ring class field.

Conjecture 4.22. Let $A / \mathbb{Q}$ be a modular abelian variety, $K$ be an imaginary quadratic field, $\chi$ be an anticyclotomic character factoring through the ring class field $H_{c}$ of $K$ of conductor $c \geq 1$, and suppose that the cardinality of $\Sigma(A, \chi)$ is odd. Let $B$ denote the indefinite quaternion algebra of discriminant $\Delta$ equal to the product of the finite primes in $\Sigma(A, \chi)$, and $f$ be the newform associated with $A$. Suppose that $f$ is primitive and has $p$-minimal Artin conductor, for all primes $p$. Then, there exists an open compact subgroup $U$ in $\hat{B}^{\times}$equipped with a surjective morphism $J_{U} \rightarrow A$ and such that the set of Heegner points in $X_{U}\left(H_{c}\right)$ is non-empty.

As a variant of the above conjecture, one can ask if we can take $U=\hat{\mathcal{R}}^{\times}$for some global order $\mathcal{R}$ in $B$. This conjecture is inspired by Corollary 4.19; we only point out that the relevant part in this conjecture is to show the existence of suitable open compact subgroups (not necessarily arising from global orders) so that we have a good understanding of rationality questions of points arising from embeddings $K \hookrightarrow B$. This would allow us to solve cases excluded by Assumption 4.9 and discuss Examples 4.20 and 4.21 above.

\section{References}

[AL] A. O. L. Atkin And W.-C. W. Li, Twists of newforms and pseudo-eigenvalues of $W$-operators, Invent. Math. 48(3) (1978), 221-243. DOI : $10.1007 /$ BF01390245. 
[BD1] M. Bertolini and H. Darmon, Heegner points on MumfordTate curves, Invent. Math. 126(3) (1996), 413-456. DOI : 10.1007 /s002220050105.

[BD2] M. Bertolini And H. Darmon, Heegner points, $p$-adic $L$-functions, and the Cerednik-Drinfeld uniformization, Invent. Math. 131(3) (1998), 453-491. DOI: 10.1007/s002220050211.

[BD3] M. Bertolini And H. Darmon, $p$-adic periods, $p$-adic $L$-functions, and the $p$-adic uniformization of Shimura curves, Duke Math. J. 98(2) (1999), 305-334. DOI: 10.1215/S0012-7094-9909809-5.

[BLR] N. Boston, H. W. Lenstra, Jr., and K. A. Ribet, Quotients of group rings arising from two-dimensional representations, C. R. Acad. Sci. Paris Sér. I Math. 312(4) (1991), $323-328$.

[Brz1] J. Brzezinski, A combinatorial class number formula, J. Reine Angew. Math. 1989(402) (1989), 199-210. DOI: 10.1515/crll. 1989.402.199.

[Brz2] J. BrZEZINSKI, On embedding numbers into quaternion orders, Comment. Math. Helv. 66(1) (1991), 302-318. DOI: 10.1007/ BF02566649.

[CCL] L. Cai, Y. Chen, and Y. Liu, Heegner points on modular curves, Trans. Amer. Math. Soc. 370(5) (2018), 3721-3743. DOI: 10.1090/tran/7053.

[Car] H. CARAYOL, Représentations cuspidales du groupe linéaire, Ann. Sci. École Norm. Sup. (4) 17(2) (1984), 191-225. DOI: 10.24033/asens. 1470 .

[Che] I. Chen, The jacobians of non-split Cartan modular curves, Proc. London Math. Soc. (3) 77(1) (1998), 1-38. DoI: 10.1112 /S0024611598000392.

[CV] C. Cornut and V. Vatsal, Nontriviality of Rankin-Selberg $L$-functions and CM points, in: "L-Functions and Galois Representations", London Math. Soc. Lecture Note Ser. 320, Cambridge Univ. Press, Cambridge, 2007, pp. 121-186. DOI: 10.1017/CB09780511721267.005.

[Dar] H. Darmon, Integration on $\mathscr{H}_{p} \times \mathscr{H}$ and arithmetic applications, Ann. of Math. (2) 154(3) (2001), 589-639. DOI: $10.2307 / 3062142$.

[Del1] P. Deligne, Travaux de Shimura, in: "Séminaire Bourbaki 1970/71 Exposés 382-399", Lecture Notes in Math. 244, Springer, Berlin, 1971, pp. 123-165. DOI: 10.1007/BFb0058700. 
[Del2] P. Deligne, Les constantes des équations fonctionnelles des fonctions L, in: "Modular Functions of One Variable, II" (Proc. Internat. Summer School, Univ. Antwerp, Antwerp, 1972), Lecture Notes in Math. 349, Springer, Berlin, 1973, pp. 501-597. DOI : $10.1007 / 978-3-540-37855-6 \_7$.

[dSE] B. De Smit And B. Edixhoven, Sur un résultat d'Imin Chen, Math. Res. Lett. 7(2) (2000), 147-153. DOI: 10.4310/MRL. 2000. v7.n2.a1.

[Edi] B. Edixhoven, On a result of Imin Chen, Preprint (1996). arXiv:alg-geom/9604008.

[Gel] S. S. GelBART, "Automorphic Forms on Adèle Groups", Annals of Mathematics Studies 83, Princeton University Press, Princeton, N.J.; University of Tokyo Press, Tokyo, 1975.

[Gro] B. H. Gross, Local orders, root numbers, and modular curves, Amer. J. Math. 110(6) (1988), 1153-1182. DOI: 10.2307/237 4689.

[GZ] B. H. Gross And D. B. ZAGIER, Heegner points and derivatives of $L$-series, Invent. Math. 84(2) (1986), 225-320. DOI: 10.1007/BF01388809.

[Hid] H. HIDA, On abelian varieties with complex multiplication as factors of the jacobians of Shimura curves, Amer. J. Math. 103(4) (1981), 727-776. DOI: $10.2307 / 2374049$.

[Hij] H. HiJikata, Explicit formula of the traces of Hecke operators for $\Gamma_{0}(N)$, J. Math. Soc. Japan 26(1) (1974), 56-82. DOI: 10.2969/jmsj/02610056.

[HPS1] H. Hijikata, A. Pizer, And T. Shemanske, Orders in quaternion algebras, J. Reine Angew. Math. 1989(394) (1989), 59-106. DOI: 10.1515/crll.1989.394.59.

[HPS2] H. Hijikata, A. Pizer, And T. Shemanske, The basis problem for modular forms on $\Gamma_{0}(N)$, Mem. Amer. Math. Soc. 82(418) (1989), 159 pp. DOI: 10.1090/memo/0418.

[KP1] D. Kohen And A. PACETTI, Heegner points on Cartan nonsplit curves, Canad. J. Math. 68(2) (2016), 422-444. DOI: 10.4153/CJM-2015-047-6.

[KP2] D. Kohen AND A. PACETTi, On Heegner points for primes of additive reduction ramifying in the base field, Trans. Amer. Math. Soc. 370(2) (2018), 911-926. DOI: 10.1090/tran/6990.

[Kol] V. A. Kolyvagin, The Mordell-Weil and Shafarevich-Tate groups for Weil elliptic curves, (Russian), Izv. Akad. Nauk SSSR Ser. Mat. 52(6) (1988), 1154-1180, 1327; translation in: Math. USSR-Izv. 33(3) (1989), 473-499. 
[Lem] S. LEMURELL, Quaternion orders and ternary quadratic forms, Preprint (2011). arXiv:1103.4922.

[Mil1] J. S. Milne, Canonical models of (mixed) Shimura varieties and automorphic vector bundles, in: "Automorphic Forms, Shimura Varieties, and L-Functions, Vol. I" (Ann Arbor, MI, 1988), Perspect. Math. 10, Academic Press, Boston, MA, 1990, pp. $283-414$.

[Mil2] J. S. MiLne, The points on a Shimura variety modulo a prime of good reduction, in: "The Zeta Functions of Picard Modular Surfaces", Univ. Montréal, Montreal, QC, 1992, pp. 151-253.

[Nek1] J. Nekovář, The Euler system method for CM points on Shimura curves, in: "L-Functions and Galois Representations", London Math. Soc. Lecture Note Ser. 320, Cambridge Univ. Press, Cambridge, 2007, pp. 471-547. DOI: 10.1017/CB09780511 721267.014.

[Nek2] J. NEKOVÁř, Eichler-Shimura relations and semi-simplicity of étale cohomology of quaternionic Shimura varieties. Ann. Sci. Éc. Norm. Supér. (4) (to appear).

[Pac] A. PACETTi, On the change of root numbers under twisting and applications, Proc. Amer. Math. Soc. 141(8) (2013), 2615-2628. DOI : $10.1090 /$ S0002-9939-2013-11532-7.

[Piz1] A. Pizer, On the arithmetic of quaternion algebras. II, J. Math. Soc. Japan 28(4) (1976), 676-688. DOI: $10.2969 / j m s j / 02840676$.

[Piz2] A. Pizer, Theta series and modular forms of level $p^{2} M, C o m$ positio Math. 40(2) (1980), 177-241.

[Shi] G. Shimura, Construction of class fields and zeta functions of algebraic curves, Ann. of Math. (2) 85(1) (1967), 58-159. DOI: 10.2307/1970526.

[Tei] J. Teitelbaum, Geometry of an étale covering of the $p$-adic upper half plane, Ann. Inst. Fourier (Grenoble) 40(1) (1990), 68-78. DOI: 10.5802/aif.1203.

[Tun] J. B. TunnelL, Local $\epsilon$-factors and characters of GL(2), Amer. J. Math. 105(6) (1983), 1277-1307. DOI: 10.2307/2374441.

[Vig] M.-F. VignÉRAs, "Arithmétique des Algèbres de Quaternions", Lecture Notes in Mathematics 800, Springer, Berlin, 1980. DOI: 10.1007/BFb0091027.

[YZZ] X. YuAn, S.-W. Zhang, AND W. Zhang, "The Gross-Zagier Formula on Shimura Curves", Annals of Mathematics Studies 184, Princeton University Press, Princeton, NJ, 2013.

[Zha] S. Zhang, Heights of Heegner points on Shimura curves, Ann. of Math. (2) 153(1) (2001), 27-147. DOI : 10.2307/2661372. 
Matteo Longo:

Dipartimento di Matematica

Università di Padova

Padova

Italy

E-mail address: mlongo@math.unipd.it

$U R L$ : www.math.unipd.it/ mlongo

Víctor Rotger:

Departament de Matemàtiques

Universitat Politècnica de Catalunya

Barcelona

Spain

E-mail address: victor.rotger@upc.edu

$U R L$ : https://www-ma2.upc.edu/vrotger

Carlos de Vera-Piquero:

Fakultät für Mathematik

Universität Duisburg-Essen

Essen

Germany

E-mail address: carlos.de-vera-piquero@uni-due.de

$U R L:$ https://www. uni-due.de/ adf538d

Primera versió rebuda el 19 de desembre de 2016, darrera versió rebuda el 18 d'abril de 2017. 